\title{
Properties of discrete X-ray sources in the starburst spiral galaxy M 83
}

\begin{abstract}
R. Soria and K. Wu
Mullard Space Science Laboratory, University College London, Holmbury St Mary, Surrey RH5 6NT, UK

e-mail: Roberto.Soria@mssl.ucl.ac.uk; kw@mssl.ucl.ac.uk

Received 3 December 2002 / Accepted 2 July 2003

Abstract. We have identified 127 discrete sources in a Chandra ACIS observation of M 83, with a detection limit of $\approx 3 \times$ $10^{36} \mathrm{erg} \mathrm{s}^{-1}$ in the $0.3-8.0 \mathrm{keV}$ band. We discuss the individual X-ray spectral and time-variability properties of $\approx 20$ bright sources with luminosities $\gtrsim 10^{38} \mathrm{erg} \mathrm{s}^{-1}$, and the statistical properties of the whole sample. About one third of the bright sources show X-ray spectra with a blackbody component at temperatures $\lesssim 1 \mathrm{keV}$, plus a powerlaw component with $\Gamma \approx 2.5$, typical of X-ray binaries in a soft state; another third have powerlaw spectra with $\Gamma \approx 1.5$, consistent with X-ray binaries in a hard state. Two bright sources show emission lines on a hard powerlaw continuum, and are probably X-ray binaries surrounded by a photo-ionized nebula or stellar wind. Among the other bright sources, we also identified two supernova remnant candidates, with optically-thin thermal plasma spectra at temperatures $\sim 0.5 \mathrm{keV}$. The two brightest supersoft sources have blackbody temperatures $k T \approx 70 \mathrm{eV}$ and luminosities $\sim 10^{38} \mathrm{erg} \mathrm{s}^{-1}$. Two candidate X-ray pulsars are detected with periods $\approx 200 \mathrm{~s}$. One $\mathrm{X}$-ray source corresponds to the core of a background FRII radio galaxy. The discrete sources can be divided into three groups, based on their spatial, color and luminosity distributions. The first group comprises supersoft sources with no detected emission above $1 \mathrm{keV}$ and blackbody spectra at temperatures $<100 \mathrm{eV}$. The second group consists of soft sources with little or no detected emission above $2 \mathrm{keV}$. They are strongly correlated with $\mathrm{H} \alpha$ emission in the spiral arms and starburst nucleus, tracing a young population. Their relative abundance depends on the current level of star-forming activity in the galaxy. Most of them are likely to be supernova remnants. The sources in the third group are mostly X-ray binaries, reaching higher X-ray luminosities than sources in the other two groups. Being a mixture of old low-mass and young high-mass systems, the whole group appears to be of intermediate age when correlated with the $\mathrm{H} \alpha$ emission. The color-color diagrams allow us to distinguish between sources in a soft and hard state.
\end{abstract}

Key words. galaxies: individual: M 83 (=NGC 5236) - galaxies: spiral - galaxies: starburst - X-rays: binaries X-rays: galaxies

\section{Introduction}

M 83 (NGC 5236) is a nearby spiral galaxy (Hubble type $\mathrm{SAB}(\mathrm{s}) \mathrm{c})$ with a circum-nuclear starburst. It is oriented almost face-on, with an inclination $i=24^{\circ}$ (Talbot et al. 1979). Its distance was estimated to be $8.9 \mathrm{Mpc}$ by Sandage \& Tamman (1987), but a value of $3.7 \mathrm{Mpc}$ was obtained more recently by de Vaucouleurs et al. (1991). A distance $\$ 5 \mathrm{Mpc}$ would place M 83 in the Centaurus A group, whose galaxies have high velocities and a large spread in morphology (de Vaucouleurs 1979; Côté et al. 1997), suggesting that the group is not yet virialized, and that merging and tidal interactions may be frequent occurrences among its members. Here and hereafter, we adopt the distance estimate of $3.7 \mathrm{Mpc}$.

M 83 has two grand-design spiral arms with ongoing star formation (e.g., Talbot et al. 1979; Tilanus \& Allen 1993). In addition, it has an even more active nuclear region $\left(\leqslant 10^{\prime \prime}\right.$ in

Send offprint requests to: $\mathrm{R}$. Soria,

e-mail: rs1@mssl.ucl.ac.uk radius) which is currently undergoing a violent starburst (Gallais et al. 1991; Elmegreen et al. 1998; Harris et al. 2001). The star formation rate due to the circum-nuclear starburst is estimated to be $\approx 0.1-0.2 M_{\odot} \mathrm{yr}^{-1}$ (de Vaucouleurs et al. 1983; Harris et al. 2001), similar to the rate of star formation in the rest of the galaxy (e.g., Buat et al. 2002). It was suggested that intense star formation may have been induced by the last close encounter with the dwarf S0 NGC 5253 (Bohlin et al. 1983; van den Bergh 1980).

M 83, together with M 82, NGC 253 and NGC 4395, accounts for about $25 \%$ of today's total rate of star formation within a distance of $10 \mathrm{Mpc}$ from the Milky Way (Heckman 1998). Massive stars are progenitors of supernova remnants (SNRs) and X-ray binaries (XRBs), the brightest non-nuclear $\mathrm{X}$-ray sources in galaxies. Observations have shown that the $\mathrm{X}$-ray properties of normal galaxies are closely related to their recent star formation activity. Galaxies with active star formation (e.g., M 82, Matsumoto et al. 2001; NGC 4038/4039, Fabbiano et al. 2001) have a large population of bright discrete 
sources, but quiescent galaxies (e.g., M 31, Supper et al. 2001; Shirey et al. 2001) are often deficient in very bright sources. Moreover, the spatial distribution of the sources is different in different galactic components. In the spiral galaxy M 81 the galactic disk contains more bright sources than the bulge (e.g. Tennant et al. 2001; Swartz et al. 2002, 2003), and the brighter sources in the disk tend to be located closer to the spiral arms (Swartz et al. 2003). For these reasons, the X-ray source population is a probe of the recent star formation activity and dynamics of the host galaxy (Wu 2001; Wu et al. 2003; Prestwich 2001; Prestwich et al. 2003), and high spatial resolution X-ray observations of M 83 are particularly important in this context.

M 83 was observed in the X-ray band by Chandra on 2000 April 29. The galaxy had also been observed by Einstein in 1979-1981 (Trinchieri et al. 1985), by GINGA in 1988 (Ohashi et al. 1990), by ROSAT in 1992-1994 (PSPC, Ehle et al. 1998; HRI, Immler et al. 1999), and by ASCA in 1994 (Okada et al. 1997). Thirteen "discrete" sources were found in the ROSAT PSPC image, and 37 with the HRI. The starburst nuclear region was unresolved in the ROSAT images. Our preliminary analysis of the Chandra data identified a total of 81 sources detected with signal-to-noise ratio $>3.5$, and resolved the ROSAT nuclear source into at least 15 discrete sources embedded in strong diffuse emission (Soria \& Wu 2002). Here we report more comprehensive results of our individual and statistical analysis of the discrete source population. We compile a more complete list of the sources detected in the ACIS-S3 chip, and determine the spectral properties of the brightest individual objects. We also quantify several statistical properties of the discrete sources and compare some of them with those found for the M 81 source population.

We organize the paper as follows. In Sect. 2 we describe the general source extraction and data analysis procedures; in Sects. 3 and 4 we present the results of our spectral and timing analysis of the brightest sources. The statistical properties of the discrete source population and the comparison with M 81 are discussed in Sect. 5.

\section{Source detection}

The Chandra observation analyzed here was carried out on 2000 April 29 (ObsID: 793), with the ACIS-S3 chip at the focus. We retrieved the data from the Chandra X-ray Center archives. The total exposure time was $50.978 \mathrm{ks}$; after screening out observational intervals with strong background flares, we retained a good time interval of $50.851 \mathrm{ks}$ (using less stringent background rejection criteria compared to Soria \& Wu 2002).

We analyzed the data of the S3 chip with the CXC software CIAO version 2.2.1. The source-finding routine wavdetect was used to identify the discrete sources in the full $(0.3-8.0 \mathrm{keV})$ band, and we calculated an exposure map at $1.0 \mathrm{keV}$ for correcting the net count rates. We also compared the lists of sources obtained from the two routines wavdetect and celldetect (the latter was used in Soria \& Wu 2002) and found only very small differences. We chose to adopt here the positions and count rates obtained with wavdetect. We first divided the $0.3-8.0 \mathrm{keV}$ band into three narrower bands: the "soft" (0.3-1.0 keV) band, the "medium" (1.0-2.0 keV) band and the "hard" (2.0-8.0 keV) band. We then produced Filtered Event Files for the three energy bands and applied wavdetect to each narrow-band image using exposure maps at $0.7,1.5$ and $4.0 \mathrm{keV}$ respectively. Each wavdetect run produced a source list with slightly different positions: we examined and compared the position of each source in the four lists to identify the corresponding objects. In all cases in which the same source was detected in more than one band, the positional difference was always $<1^{\prime \prime}$. We listed the coordinates obtained from the full band in Table A.1 (uncertainty of $\approx 0 . ' 5$ ). In the same table, we also listed the net counts in the full, soft, medium and hard bands.

We identified 127 discrete sources in the S3 chip with a signal to noise ratio $S / N>3$ in the full band. Some of these sources are not found by wavdetect in one or more narrow bands, even when we lower the detection threshold: in those cases, the net counts were estimated from an individual spectral analysis of each source (spectra extracted with psextract). When the net count values are given in brackets in Table A.1, the source has $S / N<3$ in that band.

The $90 \%$ uncertainty in the Chandra positions is 0.' 6 . To improve the absolute positions of the sources, we looked for cross-correlations with sources in the International Celestial Reference Frame (Ma et al. 1998), the Tycho 2 catalogue, the 2MASS survey, the Hubble Space Telescope (HST) Guide Star Catalogue and the US Naval Observatory Precision Measuring Machine Catalogue. However, none of those sources could be identified with sources in the Chandra ACIS-S3 image. We also compared the position of the nuclear X-ray source with the optical/UV position of the IR nucleus deduced from the HST WFPC2 archival observations. We found that a shift of 0.5 to the Chandra coordinates could align the X-ray and optical/UV positions of the nucleus. However, the WFPC2 coordinates themselves have also uncertainties of 0.5 .

We used the results of the Chandra 1Ms Deep Field observations (Rosati et al. 2002) to estimate the fraction of background sources. We obtained that $20 \pm 4$ of our sources $(\approx 15 \%)$ should be background AGN. The background contribution to sources brighter than 50 total counts $\left(\approx 0.001 \mathrm{cts} \mathrm{s}^{-1}\right)$ is expected to be $7 \pm 3$; to sources brighter than 100 total counts $\left(\approx 0.002 \mathrm{cts} \mathrm{s}^{-1}\right): 3 \pm 1$; to sources brighter than 200 total counts $\left(\approx 0.004\right.$ cts s$\left.^{-1}\right): 1 \pm 0.5$.

Finally, we obtained a smoothed, grayscale X-ray image, plotted in Fig. 1 with its coordinate grid, the $D_{25}$ ellipse and the S3 chip boundary ${ }^{1}$. A close-up view of the starburst nuclear region is shown in Fig. 2. We also show a "true-color" image (Fig. 3), obtained from adaptively-smoothed images in the soft, medium and hard bands (CIAO task csmooth with minimal significance of the signal under the Gaussian kernel $=3$ and maximal significance $=5$ ).

\footnotetext{
This image includes the most luminous source inside the $D_{25}$ ellipse, located at RA (2000) $=13^{\mathrm{h}} 37^{\mathrm{m}} 19^{\prime} .8$, Dec $(2000)=$ $-29^{\circ} 53^{\prime} 49^{\prime \prime}$, i.e., $\approx 4^{\prime} .6$ south-east of the nucleus. It has a net count rate of $0.048 \mathrm{cts} \mathrm{s}^{-1}$ and a luminosity $\approx 10^{39} \mathrm{erg} \mathrm{s}^{-1}$. However, it is located in chip S2 (just outside the S3 chip boundary), and therefore is not discussed in this paper. See Soria \& Wu (2002).
} 


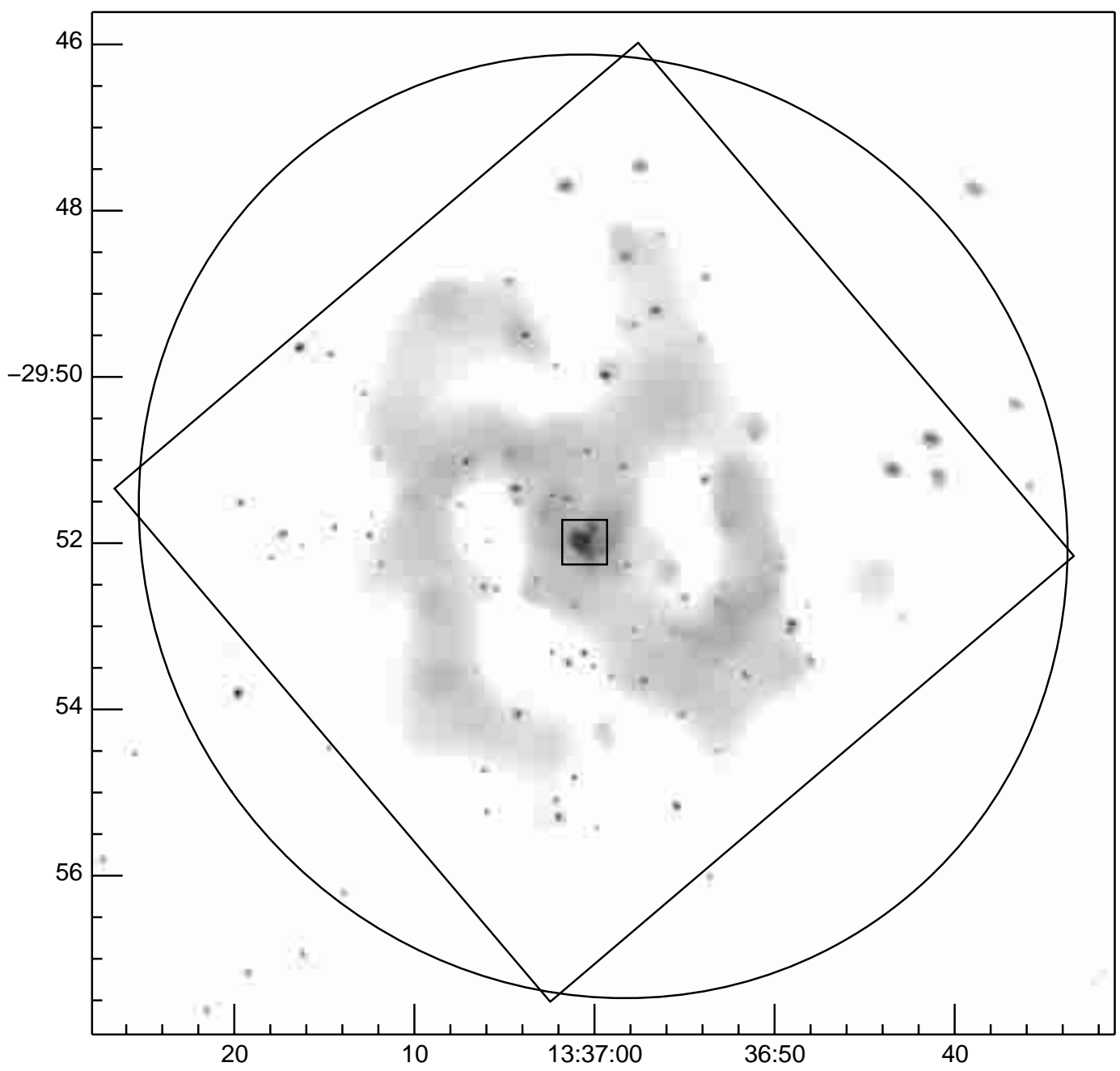

Fig. 1. Adaptively-smoothed greyscale Chandra ACIS image of M 83, in the $0.3-8.0 \mathrm{keV}$ band (square-root scale, arbitrary zeropoint). The $D_{25}$ ellipse and the $\mathrm{S} 3$ chip boundaries are overplotted. The region inside the central square is shown in greater detail in Fig. 2.

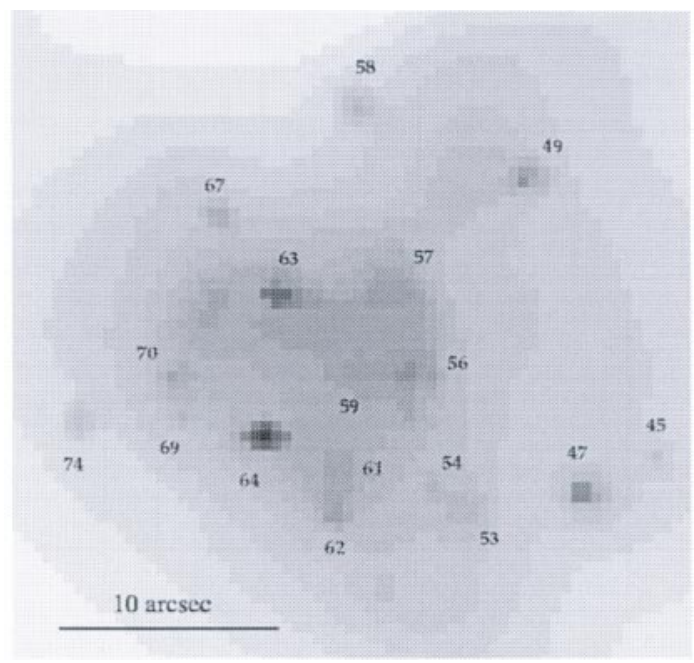

Fig. 2. Close-up view of the starburst nucleus. The source numbers refer to Table A.1. North is up, and East is left. (This figure is available in color in electronic form.)

\section{Spectral properties of individual sources}

We modelled the spectra with higher $S / N$ : about twenty sources are detected with $\gtrsim 300$ counts in the $0.3-8.0 \mathrm{keV}$ band, and two other bright, soft sources have fluxes $>100$ counts in the $0.3-1.0 \mathrm{keV}$ band alone. We also modelled the spectra of two fainter $(\sim 150$ counts) sources because of their interesting physical nature (an X-ray pulsar and a background radio galaxy, see Sects. 3.5 and 4). For sources that are not bright enough for meaningful spectral modelling, we only determined their colors and color ratios.

We fixed the redshift $z=0.00172$ and the foreground Galactic absorption column density $n_{\mathrm{H}}=4.0 \times 10^{20} \mathrm{~cm}^{-2}$ (Predehl \& Schmitt 1995; Schlegel et al. 1998), but we left the hydrogen column density component within M83 as a free parameter ${ }^{2}$. We first considered simple one-component models in XSPEC (Arnaud 1996): powerlaw, blackbody, diskblackbody and Raymond-Smith (for optically-thin thermal plasma). In most cases, they gave acceptable fits; however, a few sources required more complex spectral models. For sources suspected to be accreting compact objects, we also considered the comptonization model bmc. Spectra showing emission-line features (from candidate SNRs or

\footnotetext{
${ }^{2}$ We were also aware of the time-dependent degradation of the ACIS Quantum Efficiency at soft energies. However, we checked that this effect is still negligible for our 2000 April observation.
} 


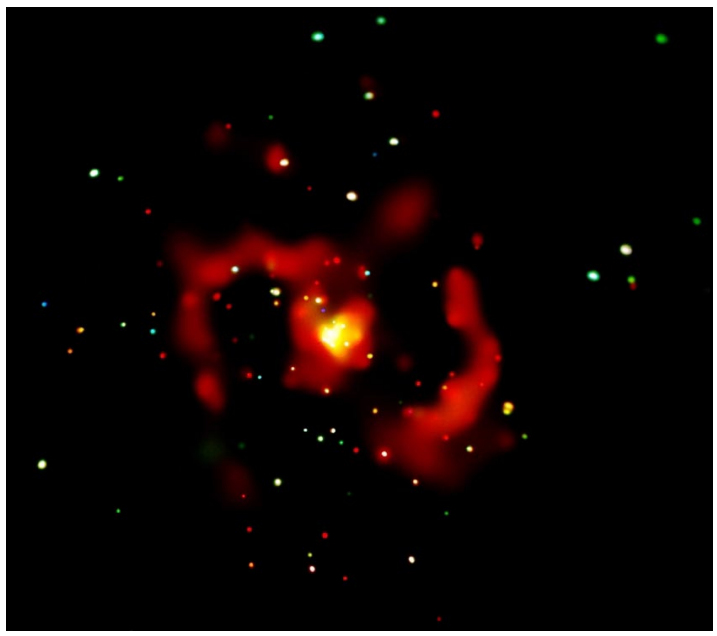

Fig. 3. A "true-colour" Chandra ACIS image of M 83 shows about 130 discrete sources (down to a luminosity of $\approx 3 \times 10^{36} \mathrm{erg} \mathrm{s}^{-1}$ for the assumed distance of $3.7 \mathrm{Mpc}$ ) and diffuse emission in the starburst nucleus and along the spiral arms. The colors are: red for $0.3-$ $1.0 \mathrm{keV}$; green for $1.0-2.0 \mathrm{keV}$; blue for $2.0-8.0 \mathrm{keV}$. The size of the image is $12^{\prime} \times 10^{\prime}$. North is up, East is left. (This figure is available in color in electronic form.)

wind-accreting XRBs), were also fitted with Gaussian lines in addition to a smooth continuum.

In total, we modelled the spectra of 23 bright sources. For convenience in our discussion, we have grouped them according to their general spectral properties and possible physical interpretation. A sample of the fitted spectra are shown in this section, the others are shown in Appendix B. The fit parameters of all 23 sources are tabulated in Appendix B.

\subsection{Nuclear source}

The presence of two galactic nuclei, each with a mass of about $1.3 \times 10^{7} M_{\odot}$, was inferred from stellar kinematic data (Thatte et al. 2000). One is a strong IR and optical source; the other is not visible at either wavelength. We have identified an X-ray point source (No. 63, see Appendix A) with a position almost coincident with that of the IR/optical nucleus (Soria \& Wu 2002). We consider this X-ray source as the galactic nucleus, though we cannot rule out the possibilities of a stellar-mass $\mathrm{X}$-ray source observed near the galactic center by chance.

The nuclear source is embedded in strong, inhomogeneous diffuse X-ray emission, which makes it difficult to subtract the background contribution, especially at low energies. However, its X-ray spectrum is well fitted by a single powerlaw (Fig. 4), with no need for an additional thermal component. We obtain a powerlaw photon index $\Gamma=1.45_{-0.24}^{+0.16}$ and an intrinsic absorption column density $n_{\mathrm{H}}=\left(1.25_{-0.93}^{+0.80}\right) \times 10^{21} \mathrm{~cm}^{-2}$, with $\chi_{v}^{2}=1.06$ for 49 d.o.f. (Table B.1). This is consistent, within the errors, with the result of our preliminary analysis in Soria \& Wu (2002). The emitted X-ray luminosity is $L_{\mathrm{x}} \approx 2.3 \times 10^{38} \mathrm{erg} \mathrm{s}^{-1}$ in the $0.3-8.0 \mathrm{keV}$ band.

The powerlaw spectrum of the nuclear source is similar to those of supermassive black holes (BHs) in active galaxies $(\Gamma \approx 1.5)$. The absence of a blackbody component in the

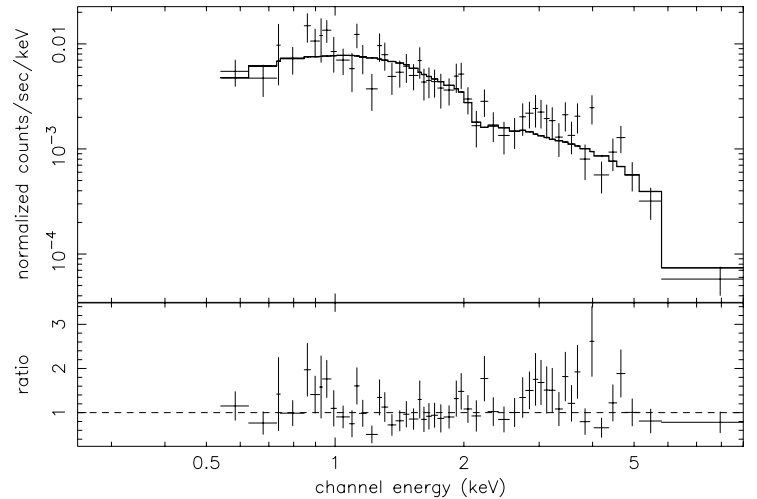

Fig. 4. Background subtracted X-ray spectral distribution (crosses), together with the best fitting absorbed power law model (histogram, upper panel), for source No. 63, coincident with the optical nucleus of M 83. The ratio of data versus model is plotted in the lower panel. Error bars along the $Y$ axis show statistical uncertainties, those along the $X$ axis represent the width of the energy bins. The X-ray spectrum is well fitted by an absorbed powerlaw with $\Gamma \approx 1.45$ (fit parameters in Table B.1).

$0.3-8.0 \mathrm{keV}$ band is consistent with a $\mathrm{BH}$ mass $\gtrsim 10^{7} M_{\odot}$, implying that the inner accretion disk has an effective blackbody temperature below $0.1 \mathrm{keV}$. The unabsorbed X-ray luminosity deduced from the fitted spectrum is comparable to the luminosity of stellar-mass accreting objects, almost ten million times below the Eddington limit of the galactic nucleus: if the source is indeed a supermassive $\mathrm{BH}$, its current accretion rate must be extremely low.

\subsection{Supersoft sources}

Two bright sources, No. 68 and 96, have detectable emission only below $1 \mathrm{keV}$, and can be classified as "supersoft". Neither was detected by ROSAT because of insufficient photon counts. We tried fitting their spectra with an absorbed blackbody, powerlaw and optically-thin thermal plasma models. The powerlaw model is inconsistent with the data, and the opticallythin plasma model requires unphysically low abundances. Both spectra are instead well fitted by a blackbody model (Fig. 5 and Table B.2). The fitted temperatures are $k T_{\mathrm{bb}}=65_{-13}^{+14} \mathrm{eV}$ for source No. 68, and $k T_{\mathrm{bb}}=58_{-28}^{+82} \mathrm{eV}$ for source No. 96 .

Most supersoft sources are believed to be accreting white dwarfs undergoing (quasi-)steady nuclear burning on their surface (van den Heuvel et al. 1992; Rappaport et al. 1994). Other models for supersoft sources include SNRs, accreting neutron stars (NSs) with large photospheres, intermediate-mass BHs, symbiotic systems, hot cores of young planetary nebulae and stripped core of tidally disrupted stars (e.g., Di Stefano \& Kong 2003). All these systems can have blackbody-like spectra with characteristic temperatures $\approx 50-80 \mathrm{eV}$. The parameters of the blackbody model for sources No. 68 and 96 are also similar to those of the supersoft sources in the nearby spirals M31 (Kahabka 1999) and M 81 (Swartz et al. 2002). 

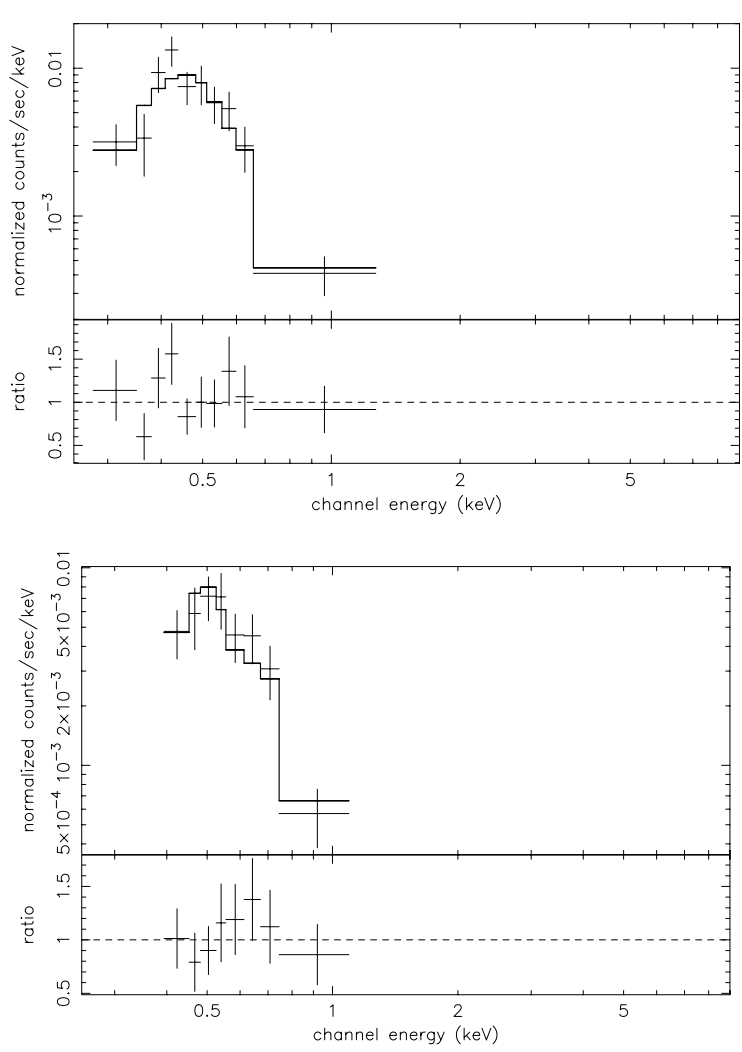

Fig. 5. The spectra of the two brightest supersoft sources (top: No. 68; bottom: No. 96); see Table B.2 for the fit parameters.

\subsection{Candidate SNRs and emission-line sources}

The spectra of sources No. 3, 8, 27 and 56 show line-like features, suggesting the possibility of emission from optically-thin thermal plasma. Fitting their spectra with a powerlaw model (Table B.3) shows that the underlying continuum is very soft for two of them (No. 8 and 56 require an unphysically high photon index $\Gamma>3.3$ ) and harder for the other two $(\Gamma \approx 1.5)$. A bremsstrahlung model (Table B.3) gives temperatures $\lesssim 1 \mathrm{keV}$ for the two soft sources, and temperatures $\gtrsim 10 \mathrm{keV}$ for the two hard ones. The same temperature range is obtained from a Raymond-Smith model. For the two softer sources, the lack of a strong Fe L-line complex requires a low metal abundance; on the other hand, this underestimates the $\mathrm{Mg}$ and $\mathrm{Si}$ emission. The $\mathrm{S} / \mathrm{N}$ is too low to allow for a meaningful fitting of different metal abundances for different elements.

Finally, we tried to model the spectra of these four sources with Gaussian lines added to a smooth continuum: we chose a powerlaw continuum for the hard sources No. 3 and 27, and a bremsstrahlung continuum for the soft sources No. 8 and 56. Four emission lines are significantly detected in source No. 3 (Table B.3): one at $E=1.32_{-0.05}^{+0.04} \mathrm{keV}$, consistent with Mg XI $(1.33 \mathrm{keV})$; one at $E=1.51_{-0.03}^{+0.04} \mathrm{keV}$, suspected to be $\mathrm{Mg}$ XII $(1.47 \mathrm{keV})$; one at $E=1.85_{-0.03}^{+0.04} \mathrm{keV}$, consistent with the Si XIII triplet $(1.84-1.87 \mathrm{keV})$; and one at $E=$ $2.60_{-0.10}^{+0.19} \mathrm{keV}$, possibly due to S XVI $(2.62 \mathrm{keV})$. Taken together, these four lines require an ionisation parameter $\log \xi \approx$ 2.5 for the emitting plasma. Some of these metal lines are also seen in sources No. 8,27 and 56. The spectral modelling and
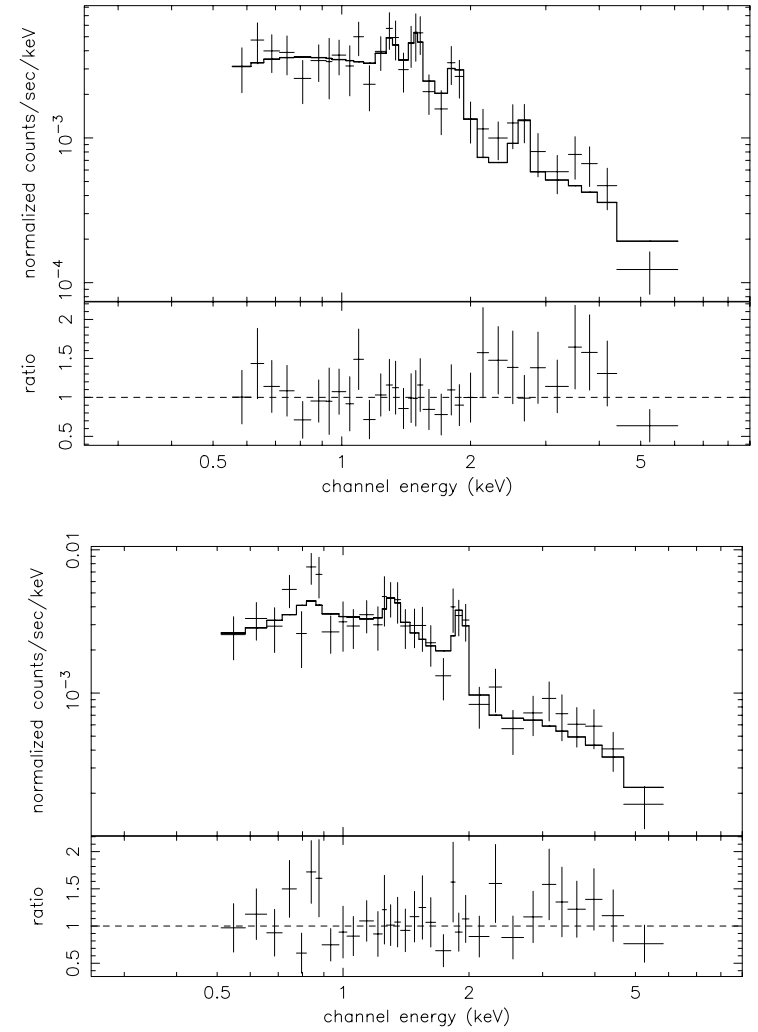

Fig. 6. Spectra of sources No. 3 (top) and 27 (bottom) together with a best-fitting absorbed power law model with emission lines (see Table B.3).

luminosity estimate for source No. 56 has the largest uncertainty, because the source is located in the nuclear starburst ring, and it is embedded in strong, non-uniform diffuse emission from hot thermal plasma. Its spectrum may also be contaminated by other nearby, unresolved sources.

Thermal plasma temperatures $\approx 0.7 \mathrm{keV}$ and the presence of emission lines from $\alpha$-elements make sources No. 8 and 56 strong SNR candidates; however, long-term timevariability studies are required to confirm this identification ${ }^{3}$.

The harder spectrum seen from sources No. 3 and 27 requires much higher plasma temperatures, or a powerlaw continuum. We cannot rule out an SNR origin for these two sources: in young SNRs, the forward shock (propagating into the circumstellar medium) produces hard X-ray emission, while the reverse shock (propagating into the ejecta) produces soft X-ray emission. If the cooling shell at the contact discontinuity is optically thick, the soft component is not detectable (Chevalier \& Fransson 1994). High-temperature optically-thin thermal

\footnotetext{
3 After completing our spectral analysis, the Chandra ACIS data of a short (good time interval of $6.4 \mathrm{ks}$ ) observation of M 83 from 2001 April have become available in the public archive. A quick-look analysis of the new dataset suggests that the flux of source No. 8 has increased by a factor of 2 . If this is confirmed, the SNR identification becomes less likely, and the thermal plasma is more likely to be ionized by an accreting XRB. A detailed comparison of all the sources' fluxes and colors in the two observations, and between the ROSAT and Chandra observations, is beyond the scope of this paper.
} 

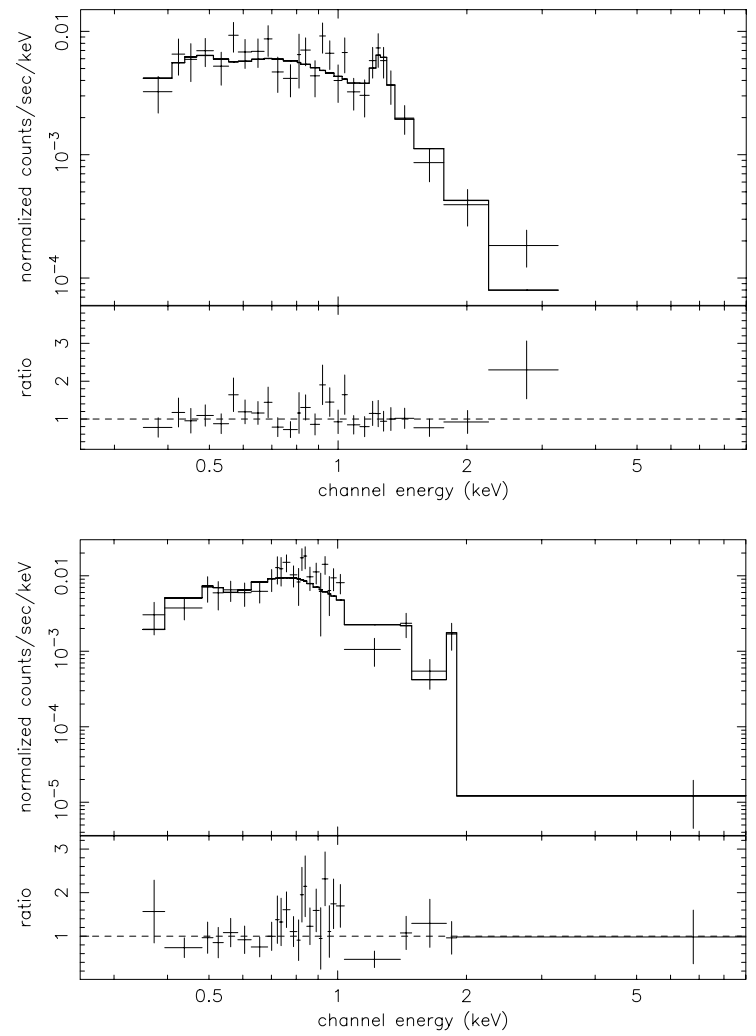

Fig. 7. Spectra of sources No. 8 (top) and 56 (bottom) together with a best-fitting single-temperature thermal plasma model (see Table B.3).

emission has been detected in some young Galactic remnants (e.g., SN 1998S, Pooley et al. 2002).

However, we note that a hard powerlaw continuum with the superposition of emission lines can originate from X-ray binaries surrounded by a photo-ionized nebula. For example, strong recombination lines are seen in the Galactic high-mass XRBs Cyg X-3, Cen X-3 and Vela X-1 (e.g., Liedahl et al. 2000 and references therein), which are accreting from highly ionized stellar winds, and reach X-ray luminosities $\sim 10^{38} \mathrm{erg} \mathrm{s}^{-1}$. (In Vela X-1, the differential emission measure suggests an ionization parameter $2 \lesssim \log \xi \lesssim 3$ similar to what is observed in source No. 3; however, the absorbing column density seen by the continuum emission is higher in Vela X-1; see Sako et al. 1999.) We suggest that source No. 3 and 27 may be such accreting systems in M 83.

No radio or optical counterparts were found for sources No. 3, 8 and 27, nor do they correspond to any of the six historical supernovae ( $\mathrm{SNe}$ ) in the galaxy ${ }^{4}$. Conversely, no $\mathrm{X}$-ray sources are detected at the positions of the five historical SNe observed in the disk. However, one of the historical SNe, type-II SN 1968L (Thackeray 1968; Wood \& Andrews 1974), is in the circum-nuclear starburst ring. Its reported optical coordinates ${ }^{5}$ are just $\approx 1^{\prime \prime}$ away from the Chandra position of source No. 56. We are unable to ascertain whether or not the X-ray source is indeed the remnant of SN 1968L. In any case, SNe occur at a rate $\sim 1$ per century in the nuclear region. With its soft

\footnotetext{
${ }^{4}$ http://cfa-www.harvard.edu/cfa/ps/lists/ Supernovae.html

${ }^{5}$ http://nedwww.ipac. caltech.edu/
}
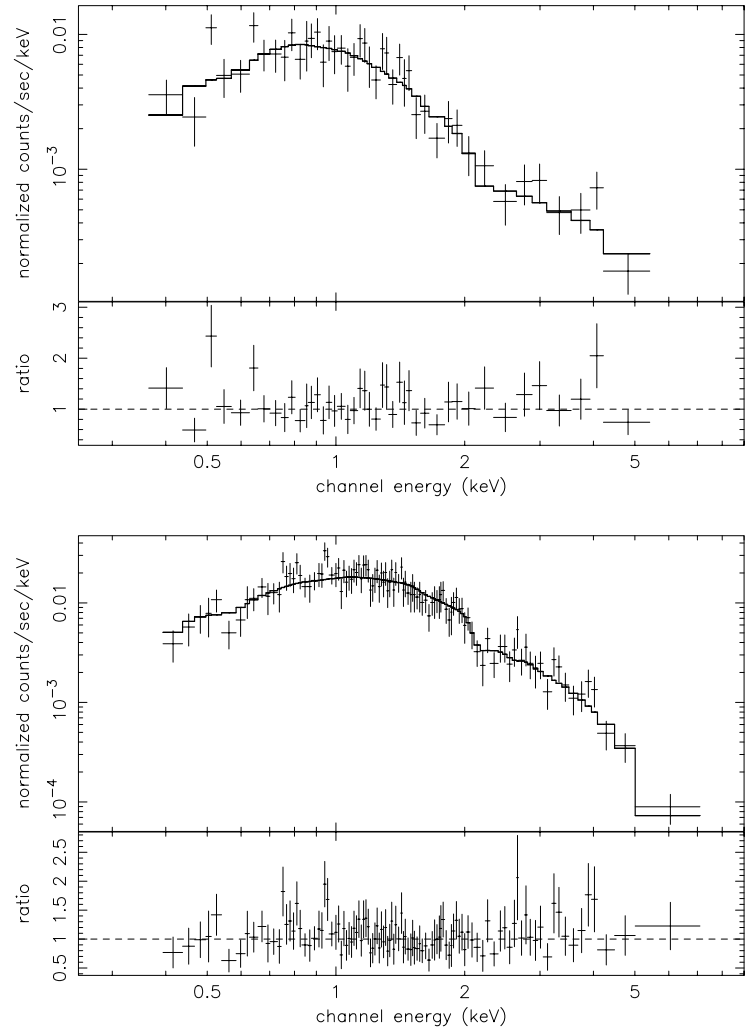

Fig. 8. Spectra of sources No. 73 (top) and 86 (bottom) together with a best-fitting absorbed power law model (see Table B.4).

spectrum, inconsistent with powerlaw models typical of accreting binaries, source No. 56 is almost certainly an X-ray SNR.

Two more sources were suggested as X-ray SNR candidates by Immler et al. (1999), based on VLA radio and ROSAT HRI data. In our Table A.1 catalogue, they are sources No. 73 and 86, corresponding to H23 and H26 in Immler et al. (1999). Source No. 73 is located in the bar, in front of the dust lane, $\approx 30^{\prime \prime}$ north-east of the nucleus, and it coincides with the position of a compact radio source (Cowan \& Branch 1985; Cowan et al. 1984). Source No. 86 is located near the end of the northeastern arm. Both sources are situated in giant H II regions.

The spectra of both sources can be fitted by singletemperature vmekal models. For source No. 73, the fit parameters are $k T_{\mathrm{vm}}=(4.2 \pm 0.7) \mathrm{keV}, n_{\mathrm{H}}=(4.8 \pm 2.0) \times 10^{20} \mathrm{~cm}^{-2}$ $\left(\chi_{v}^{2}=0.97,42\right.$ d.o.f.). For source No. 86, we obtain $k T_{\mathrm{vm}}=$ $(1.8 \pm 0.1) \mathrm{keV}, n_{\mathrm{H}}=(24 \pm 2) \times 10^{20} \mathrm{~cm}^{-2}\left(\chi_{v}^{2}=0.60\right.$, 105 d.o.f.). In both cases, the fitted temperatures are higher than for most SNRs. More importantly, their spectra are smooth, without any significant emission-line features. They could also be well fitted by powerlaw or Comptonised blackbody models, with parameters similar to those typical of XRBs in a high-soft state (Table B.4). The nature of the two sources is therefore still open to investigation. We argue that they are more likely to be XRBs rather than young SNRs. If so, an estimated emitted luminosity $\gtrsim 3 \times 10^{38} \mathrm{erg} \mathrm{s}^{-1}$ in the $0.3-8.0 \mathrm{keV}$ band makes source No. 86 a likely BH candidate.

Among the fainter X-ray sources, No. $84\left(L_{\mathrm{x}} \approx 1.5 \times\right.$ $10^{37} \mathrm{erg} \mathrm{s}^{-1}$ in the $0.3-8.0 \mathrm{keV}$ band) coincides with a flat-spectrum radio source (spectral index $0.21 \pm 0.14$; 
Cowan et al. 1994). The radio flux was $(0.39 \pm 0.05) \mathrm{mJy}$ at $20 \mathrm{~cm}$, and $(0.50 \pm 0.05) \mathrm{mJy}$ at $6 \mathrm{~cm}$ in $1990-1992$. The radio brightness at $6 \mathrm{~cm}$ faded by $(33 \pm 7) \%$ between $1983-$ 1984 and 1990-1992. The source is probably an evolving radio SNR (Cowan et al. 1994); the Chandra detection suggests that source No. 84 may be its X-ray counterpart. Unfortunately the X-ray source is not bright enough for detailed spectral modeling. Another faint source, No. $100\left(L_{\mathrm{x}} \approx 6 \times 10^{36} \mathrm{erg} \mathrm{s}^{-1}\right.$ in the $0.3-8.0 \mathrm{keV}$ band), coincides with the non-thermal radio source No. 7 in Cowan et al. (1994). The radio source faded by $\approx(25 \pm 11) \%$ at $6 \mathrm{~cm}$ between $1983-1984$ and $1990-1992$.

\subsection{X-ray binaries}

The remaining sources for which we could do meaningful spectral modelling do not show any spectral features resembling line emission from optically-thin thermal plasma, nor do they have the thermal blackbody-like soft spectra typical of supersoft sources. Most of them are likely to be XRBs containing an accreting BH or NS. An absorbed powerlaw provided a good fit for the X-ray spectra of most sources (Table B.4). From the best-fit photon indices, we notice that the sources can be divided into two groups. The first group (No. 5, 31, 33, 44, 60, 78,85 and 121) contains sources with hard spectra: all of them have photon indices $\Gamma \approx 1.5$, except for source No. 78 , which is even harder, with $\Gamma \approx 1.0$. Sources in the second group (No. 64, 72, 88, 104 and 113, plus sources No. 73 and 86 described in Sect. 3.3) have much steeper (softer) spectra, with $\Gamma>2$. Therefore, we also fitted the spectra of this second group of sources with the diskbb and bmc models in XSPEC: both provided equally acceptable or better fits than a powerlaw model. Typical fit temperatures inferred from the diskbb model are within the $0.7-1.5 \mathrm{keV}$ range. For the bmc model, we obtain fit temperatures $\sim 0.2-0.8 \mathrm{keV}$ for the seed photon component.

The luminosity and spectral parameters obtained from the powerlaw, diskbb and bmc models are similar to those found in Galactic XRBs, supporting our interpretation that most of these bright sources are accreting NSs and BHs. Galactic XRBs often have two distinguishable X-ray spectral states: the soft state, when the spectrum is dominated by a thermal (blackbody or disk-blackbody) component at a temperature $k T \sim 1 \mathrm{keV}$, sometimes with an additional powerlaw component of index $\Gamma \sim 2.5$; and the hard state, characterized by a simple powerlaw spectrum with a photon index $\Gamma \sim 1.5$. The bright sources in M83 can also be classified into two states with analogous spectral properties: roughly half of them appear to be in the soft state and half in the hard state. A photon in$\operatorname{dex} \Gamma \approx 1.0$ inferred for source No. 78 is unusually hard but not unique among XRBs: for example, the transient high-mass XRB SMC X-2 also has $\Gamma \approx 1.0$ and luminosity $\approx 10^{38} \mathrm{erg} \mathrm{s}^{-1}$ in its high state (Corbet et al. 2001).

\subsection{A background radio galaxy}

Source No. 39 is certainly identified as a background object because it is coincident with an inverted-spectrum radio source (source 3 in Cowan \& Branch 1985; Cowan et al. 1994), which is thought to be the core of an FR II radio galaxy. (This identification was also noted by Stockdale et al. 2001.) In addition to the flat-spectrum radio core, VLA $6 \mathrm{~cm}$ and $20 \mathrm{~cm}$ images also show two distinct radio lobes, at $\approx 25^{\prime \prime}$ north-west and southeast of the core (Cowan et al. 1994), The lobes are brighter than the core, and have a steep spectrum ( spectral indices $\approx-1$ ).

From the Chandra data, we estimate an emitted X-ray flux $f_{\mathrm{x}}=2.2 \times 10^{-14} \mathrm{erg} \mathrm{cm}^{-2} \mathrm{~s}^{-1}$ in the $0.5-3.0 \mathrm{keV}$ band, and $f_{\mathrm{x}}=$ $5.0 \times 10^{-14} \mathrm{erg} \mathrm{cm}^{-2} \mathrm{~s}^{-1}$ in the $0.3-8.0 \mathrm{keV}$ band. This corresponds to a monochromatic luminosity at $2 \mathrm{keV}$ (defined like in Fabbiano et al. 1984) $l_{\mathrm{x}}=5.5 \times 10^{24}\left(d_{\mathrm{L}} / 10^{9} \mathrm{pc}\right)^{2} \mathrm{erg} \mathrm{s}^{-1} \mathrm{~Hz}^{-1}$, where $d_{\mathrm{L}}$ is the luminosity distance. The monochromatic luminosity of the radio nucleus at $5 \mathrm{GHz}$ is (Cowan et al. 1985) $l_{\mathrm{RN}}=1.6 \times 10^{30}\left(d_{\mathrm{L}} / 10^{9} \mathrm{pc}\right)^{2} \mathrm{erg} \mathrm{s}^{-1} \mathrm{~Hz}^{-1}$. These two values satisfy the linear correlation for FRII galaxies and quasars in the $\left(\log l_{\mathrm{x}}, \log l_{\mathrm{RN}}\right)$ plane (Fig. 9 in Fabbiano et al. 1984), regardless of the assumed distance.

We can estimate the distance from the fact that the radio luminosity of an FRII radio galaxy is always $L_{20 \mathrm{~cm}} \gtrsim$ $10^{32} \mathrm{erg} \mathrm{s}^{-1} \mathrm{~Hz}^{-1}$, and $L_{6 \mathrm{~cm}} \gtrsim 10^{32} \mathrm{erg} \mathrm{s}^{-1} \mathrm{~Hz}^{-1}$, for a Hubble constant $H_{0}=75 \mathrm{~km} \mathrm{~s}^{-1} \mathrm{Mpc}^{-1}$ (e.g., Owen \& Ledlow 1994; Kembhavi \& Narlikar 1999). If the radio galaxy seen behind M 83 is at redshift $z=1$ (corresponding to $d_{\mathrm{L}}=4.7 \mathrm{Gpc}$ for $q_{0}=0.5$ and $\left.H_{0}=75 \mathrm{~km} \mathrm{~s}^{-1} \mathrm{Mpc}^{-1}\right)$, its monochromatic radio luminosities are $L_{20 \mathrm{~cm}}=1.5 \times 10^{32} \mathrm{erg} \mathrm{s}^{-1} \mathrm{~Hz}^{-1}$, and $L_{6 \mathrm{~cm}}=0.7 \times 10^{32} \mathrm{erg} \mathrm{s}^{-1} \mathrm{~Hz}^{-1}$ (Cowan et al. 1994). Its emitted X-ray luminosity is then $L_{\mathrm{x}}=1.3 \times 10^{44} \mathrm{erg} \mathrm{s}^{-1}$ in the $0.3-8.0 \mathrm{keV}$ band. Values of $L_{\mathrm{x}} \sim 10^{44} \mathrm{erg} \mathrm{s}^{-1}$ are typical of FRII radio galaxies. We conclude that the source is located at a redshift $z \gtrsim 1$. (We have no elements to determine an upper limit to its distance.)

The angular diameter distance is only a weak function of redshift, for $z \gtrsim 0.75$ : the inferred total size of the radio lobes projected in the plane of the sky is $\approx 250-280 \mathrm{kpc}$. This is an average value for this class of objects (Blundell et al. 1999).

\section{Variability of the sources}

We searched for variabilities of the brightest sources on timescales between 10 and $50000 \mathrm{~s}$. Shorter periods cannot be investigated, because the ACIS observation was carried out in "Timed Exposure" mode, collecting photons and reading them out every $\approx 3.2 \mathrm{~s}$. We were aware that spurious periods could be found at $\approx 707 \mathrm{~s}$ and $\approx 1000 \mathrm{~s}$ due to dithering. After extracting lightcurves with standard CIAO routines, we carried out discrete Fourier transforms to obtain the power density spectra. Null results were obtained for most sources; however, periods of $174.9 \mathrm{~s}$ and $201.5 \mathrm{~s}$ were found for sources No. 33 and 113 respectively. We checked that neither the background emission around those sources nor other bright nearby sources showed similar periodicities. Hence, we conclude that the periodic variabilities are probably intrinsic to the X-ray sources.

We folded the two X-ray lightcurves according to those periods and found large amplitude variations (Figs. 9 and 10). Their pulse amplitudes are approximately 0.5 of the mean levels, similar to those seen in Galactic X-ray pulsars. The spectra of the two sources are well fitted by a simple powerlaw (Table B.4), consistent with the spectra of typical X-ray 


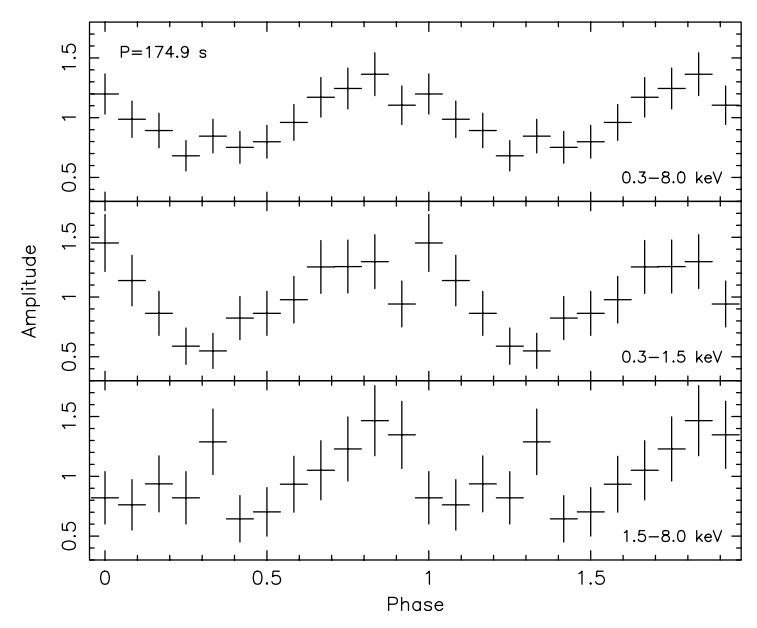

Fig. 9. Lightcurves of source No. 33 in the total, soft and hard bands, normalized to the mean count rates, folded on a period of $174.9 \mathrm{~s}$.

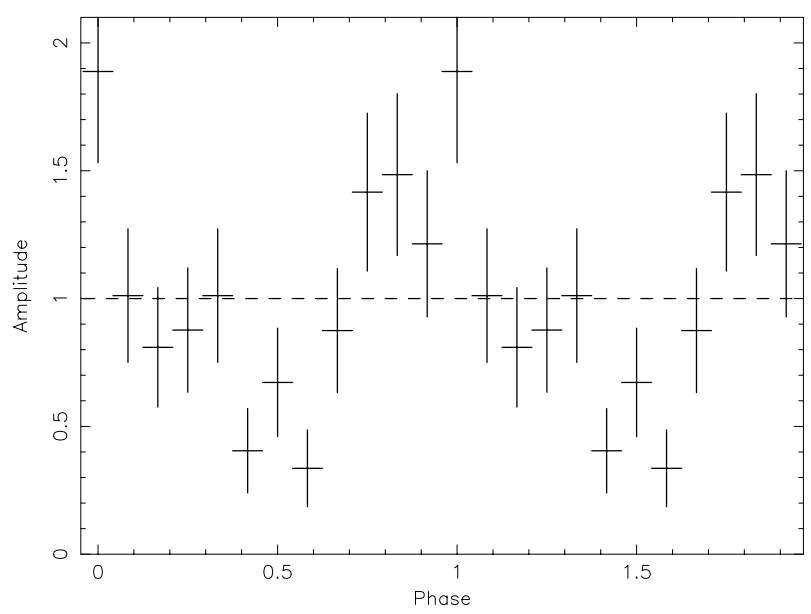

Fig. 10. Lightcurve of source No. 113, normalized to the mean count rate, folded on a period of $201.5 \mathrm{~s}$.

pulsars. These two systems may be Be XRBs (i.e., accreting NSs in eccentric orbit around Be stars) in outburst, similar to those often observed in the Magellanic Clouds (e.g., Negueruela 1998; Laycock et al. 2002). If so, repeated observations should show luminosity variations over the orbital period, which in Galactic Be XRBs is typically $\sim 100 \mathrm{~d}$ for spin periods $\sim 100 \mathrm{~s}$ (Corbet 1986).

\section{Statistical properties of the source population}

\subsection{Brightness distribution}

Among the 127 sources in the S3 chip, 28 are located in the inner disk, defined as the starburst nuclear region plus the bar, within $60^{\prime \prime}$ of the X-ray center; 17 of them are within $16^{\prime \prime}$ from the galactic center. The luminosity functions of the 28 "inner disk" sources and of the 99 "outer disk" sources differ substantially (see also Soria \& Wu 2002). Above the completeness limit ( $\approx 50$ counts for the nuclear region), the innerdisk sources have a simple powerlaw luminosity distribution with an index of -0.7 (Fig. 11). In contrast, the luminosity distribution of the other sources shows a deficiency of bright

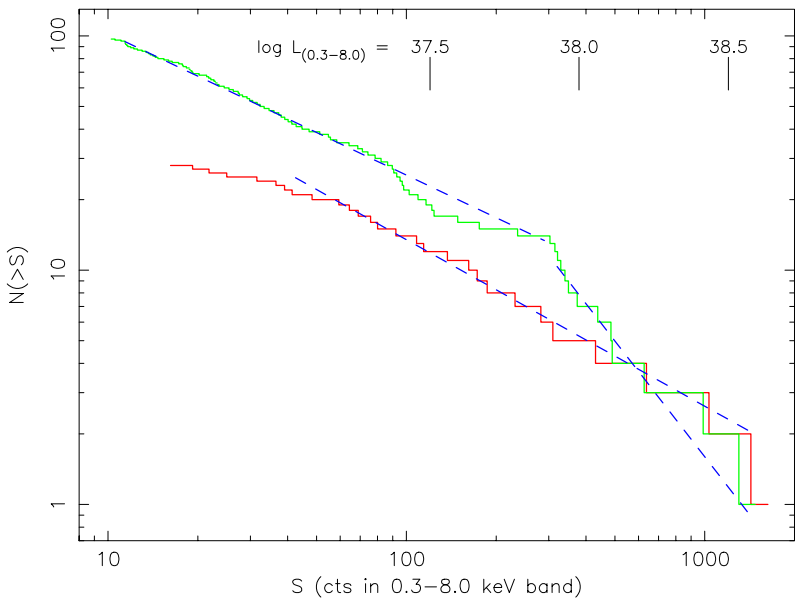

Fig. 11. Red histogram: cumulative luminosity distribution of the discrete sources in the inner region ( $d<60^{\prime \prime}$ from the galactic center), together with a powerlaw approximation (index -0.7 ). Green histogram: cumulative luminosity distribution of the sources in the outer disk, together with a broken powerlaw approximation (break at an emitted luminosity of $8 \times 10^{37} \mathrm{erg} \mathrm{s}^{-1}$ for the assumed spectral model; indices of -1.6 and -0.6 above and below the break). (This figure is available in color in electronic form.)

objects above 300 counts. For a distance of $3.7 \mathrm{Mpc}$ and an assumed absorbed powerlaw spectral model $\left(\Gamma=1.7, n_{\mathrm{H}}=\right.$ $10^{21} \mathrm{~cm}^{-2}$ ), 300 counts correspond to an emitted luminosity of $8 \times 10^{37} \mathrm{erg} \mathrm{s}^{-1}$. If we model the luminosity function with a broken powerlaw with a break at about 300 counts, we obtain powerlaw indices of -0.6 and -1.6 above and below the break. There may be another break at about 100 counts, but a more quantitative analysis is impossible because of the small number of sources. The detection limit is $\approx 12$ counts, however we estimate that the sample of outer-disk sources is complete only down to $\approx 20$ counts: a few soft sources with $\$ 20$ counts may be missed if located in star-forming regions along the spiral arms (characterised by diffuse soft emission).

The luminosity distribution of bright X-ray sources reflects the star-formation activity of the host galaxy and/or the host environments in the recent past (Wu 2001; Wu et al. 2003; Prestwich 2001; Kilgard et al. 2002). For example, there is a lack of bright sources in relatively quiescent galaxies (e.g., M31, Kong et al. 2002) while they are much more abundant in starburst (e.g., M 82, Matsumoto et al. 2001) and interacting galaxies (e.g., NGC 4038/4039, Zezas et al. 2002). Such differences can also be observed between different components of the same galaxy: for example, in M 81, the sources in the bulge show a broken-powerlaw luminosity function typical of quiescent galaxies, but the sources in the disk show a simple powerlaw luminosity function similar to those of starburst and interacting galaxies (Tennant et al. 2001; Swartz et al. 2003).

We may explain the flat powerlaw luminosity function of the nuclear sources in M 83 in terms of current starburst activity. The explanation of the luminosity function of the outer disk sources is less straightforward. Some of the sources might have been formed in the spiral arms as a result of continuous, density-wave induced star formation; others might be remnants from previous starburst epochs, perhaps due to past close encounters of M 83 with its neighbors. We speculate that this may 
explain the break-like features seen in the luminosity function between $\approx 3 \times 10^{37}$ and $10^{38} \mathrm{erg} \mathrm{s}^{-1}$. We also note that the faint end of the luminosity functions for inner and outer disk sources have similar slopes. If we interpret the faint end as the quasistationary part of the source distribution, less prone to transient perturbations such as starburst episodes, then their similarity suggests that the underlying processes determining the luminosity functions in the inner and outer disk are the same.

Whether or not some of the bright sources are transients does not affect the shape of the luminosity function, provided that the transitional probability between the high and the low states is quasi-stationary. In other words, the transient nature of the sources will not introduce features in a luminosity function unless the transitional timescale is comparable to the evolutionary timescale of the luminosity function. Many XRBs in our Galaxy show spectral transitions on a few months/years timescale, while the luminosity function should evolve on a much longer timescale.

\subsection{Spectral and color distribution}

We constructed color-color diagrams to demonstrate that the discrete sources can be classified into physically distinct groups. We considered two different definitions of the color indices: the conventional indices $[(M-S) /(M+S),(H-$ $S) /(H+S)$ ], used for example by Swartz et al. (2002) in the study of M 81 sources, and the alternative color indices $[(H-M) /(H+M+S),(M-S) /(H+M+S)]$ suggested by Prestwich et al. (2003).

We first considered the sources for which we have obtained individual spectral fits (Sect. 3), as indicators of the physical nature of the sources in the various regions of the color-color diagrams (top panels of Figs. 12 and 13). We then compared their colors with those expected from a sample of fundamental spectral models (bottom panels of Figs. 12 and 13). The spectral models that we considered are: powerlaws with photon indices $\Gamma=1.3$ and $\Gamma=1.7$ (characteristic of XRBs in the hard state); disk-blackbodies with $k T_{\text {in }}=0.5 \mathrm{keV}$ and $k T_{\text {in }}=1.0 \mathrm{keV}$ (typical of XRBs in the soft state); blackbody with $k T_{\mathrm{bb}}=0.1 \mathrm{keV}$ (supersoft sources); optically-thin, single-temperature thermal plasma at $k T_{\mathrm{rs}}=0.5 \mathrm{keV}$ (typical of SNRs). Along each model curve, the column density increases from the bottom to the top, from $n_{\mathrm{H}}=4 \times 10^{20} \mathrm{~cm}^{-2}$ (line-of-sight foreground absorption for M 83) to $n_{\mathrm{H}}=7.5 \times$ $10^{21} \mathrm{~cm}^{-2}$ for the blackbody and optically-thin thermal plasma models, and to $n_{\mathrm{H}}=2 \times 10^{22} \mathrm{~cm}^{-2}$ for the powerlaw and diskblackbody models.

Different types of sources are clearly distinguished in both color-color diagrams. With the conventional choice of color indices (Fig. 12) one can separate XRBs (with $(M-S) /(M+S) \gtrsim$ -0.2 ) from the other sources. For the XRBs, it is then possible to distinguish whether a source is intrinsically hard, or simply more absorbed. However, the alternative color indices (Fig. 13) appear to be better in separating supersoft sources, SNRs and XRBs. When we plot the colors indices of all the sources found in the S3 chip (Figs. 14 and 15), the advantage
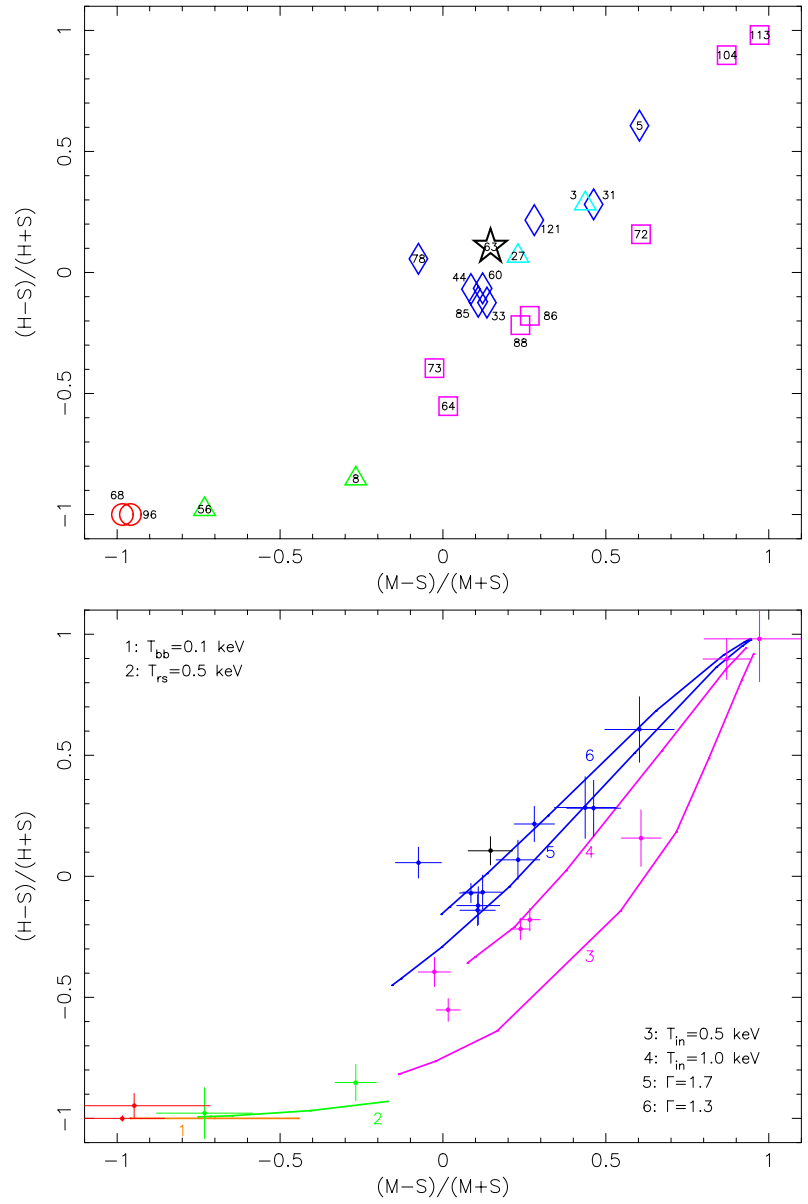

Fig. 12. Top panel: Color-color diagram of the 22 sources in M 83 (the source associated with the background radio galaxy is not included) for which individual spectral fitting is available. Sources with a featureless, hard powerlaw spectrum are plotted as blue diamonds. Sources with emission lines on top of a hard powerlaw spectrum are plotted as cyan triangles. Magenta squares are sources with a featureless soft spectrum. Green triangles are sources characterised by soft, optically-thin thermal plasma emission. Red circles are supersoft sources (blackbody-like emission at temperatures $<80 \mathrm{eV}$ ). The black star is the galactic nucleus. The numbers refer to the source catalogue in Table A.1. Bottom panel: the colors of those same 22 sources (plotted here with their error bars) are compared with those expected from some simple spectral models: blackbody, disk-blackbody, powerlaw, and optically-thin thermal plasma. The column density increases along each model curve from bottom left to top right, from $n_{\mathrm{H}}=4 \times 10^{20} \mathrm{~cm}^{-2}$ (line-of-sight foreground absorption for M 83) to $n_{\mathrm{H}}=7.5 \times 10^{21} \mathrm{~cm}^{-2}$ for the blackbody and optically-thin thermal plasma models, and to $n_{\mathrm{H}}=2 \times 10^{22} \mathrm{~cm}^{-2}$ for the powerlaw and disk-blackbody models. (This figure is available in color in electronic form.)

of the alternative choice of indices is more obvious, especially for the faint sources.

We may roughly divide the alternative color-color diagram into three regions, each dominated by a different source type. Here and hereafter we refer to the sources in those three regions as group A, B and C respectively (Fig. 15). Group A is dominated the "classical" supersoft sources, which have negligible emission in the medium and hard X-ray bands and therefore 

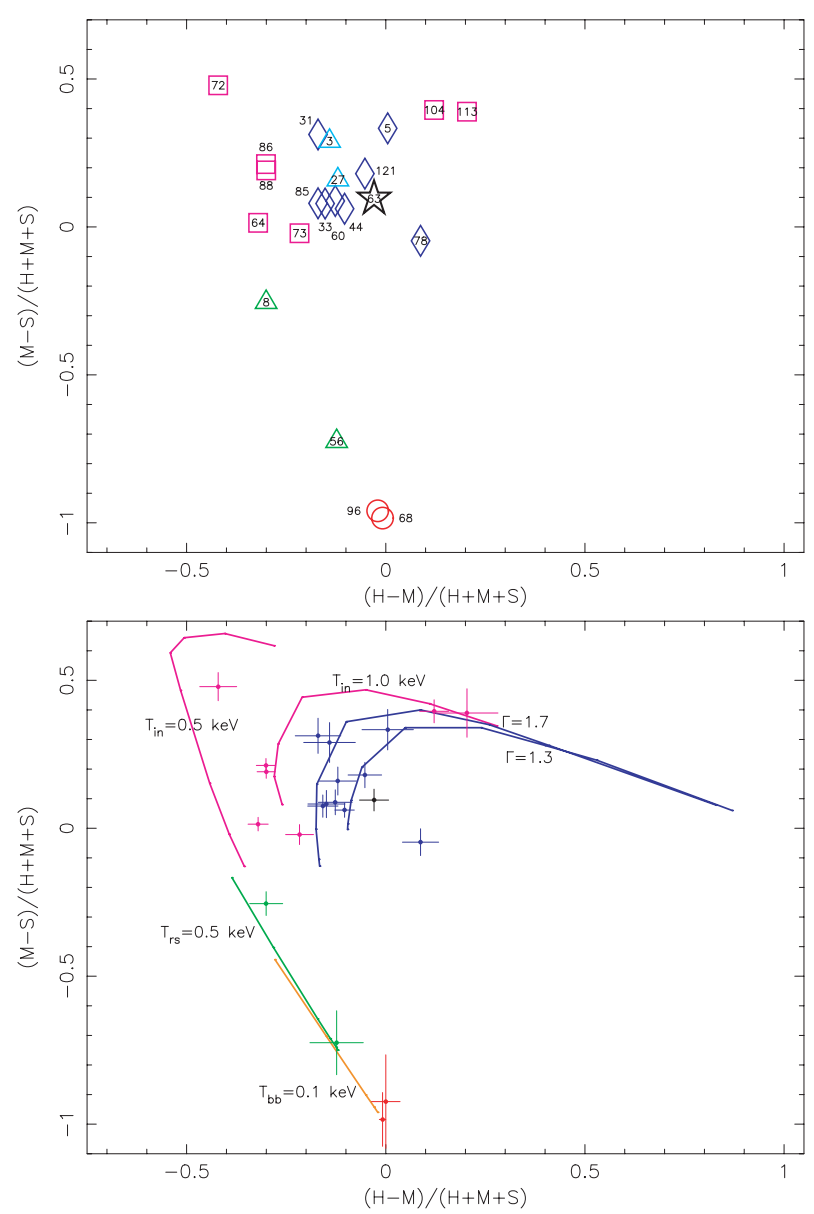

Fig. 13. Color-color diagrams for the alternative color indices. The symbols and colors of the 22 sources, and the superimposed spectral models are the same as in Fig. 12. For each spectral model, the absorbing column density increases from the bottom to the top of the representative curves.

cluster around $(0,-1)$ regardless of absorption ${ }^{6}$. Group B includes all the sources located at $-0.85 \lesssim(M-S) /(S+M+H) \lesssim$ -0.15 . Most of them are found along a straight line in the diagram, with only a narrow spread in $(H-M) /(S+M+H)$. This is consistent with emission from single-temperature opticallythin thermal plasma at $k T_{\mathrm{rs}} \approx 0.4-0.8 \mathrm{keV}$, typical of SNRs (Prestwich et al. 2003). Only a few group B sources have slightly harder spectra, consistent with emission from a twotemperature optically-thin plasma with $k T_{\mathrm{rs}, 1} \approx 0.5 \mathrm{keV}$ and $k T_{\mathrm{rs}, 2} \approx 5-10 \mathrm{keV}$. Alternatively, but less likely, they may have a composite spectrum consisting of a very absorbed, hard powerlaw and a (dominant) reprocessed soft component, as observed in some Galactic NSs (see Sect. 5.4).

Group C sources are located at $-0.15 \lessgtr(M-S) /(S+M+$ $H) \lesssim 0.6$, (Fig. 15) with a much broader spread in $(H-M) /(S+$ $M+H)$. This corresponds to a broad spread in both their absorbing column densities and their intrinsic spectral hardness. We believe that most of them are XRBs, although a few hard,

\footnotetext{
${ }^{6}$ For an operative way of identifying and classifying soft and supersoft sources in Chandra ACIS observations, see Di Stefano \& Kong (2003).
}
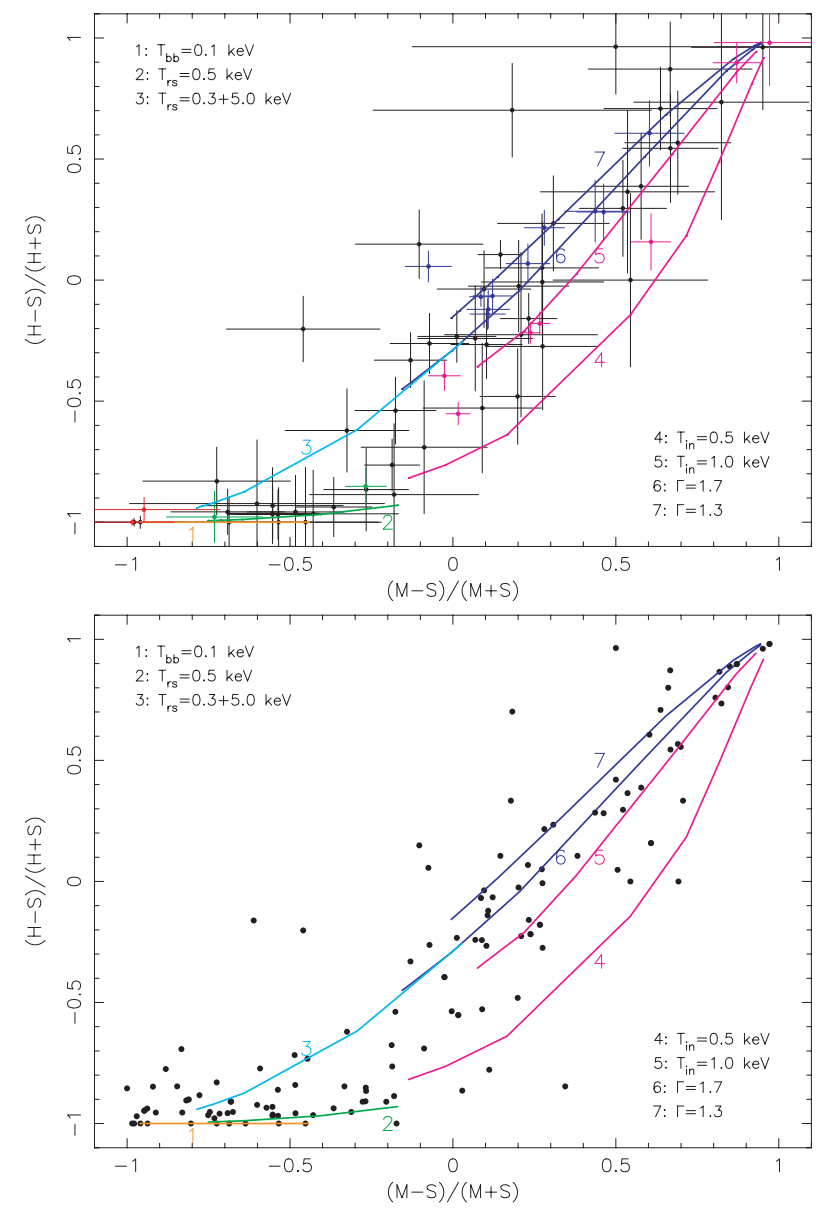

Fig. 14. Top panel: color-color diagram for sources brighter than $\approx 10^{37} \mathrm{erg} \mathrm{s}^{-1}$, with respective error bars. Bottom panel: color-color diagram for the all sources in the S3 chip, without error bars. In addition to the models shown in Fig. 12, we have also overplotted a two-temperature thermal plasma model $\left(k T_{\mathrm{rs}, 1}=0.3 \mathrm{keV}, k T_{\mathrm{rs}, 2}=\right.$ $5.0 \mathrm{keV}$ ), normalised so that the high-temperature component accounts for $25 \%$ of the emitted flux.

highly-absorbed sources may be background AGN. All sources with a disk-blackbody, disk-blackbody plus powerlaw, or simple powerlaw spectra fall in this region of the diagram.

Incidentally, we notice that the emission-line sources No. 3 and 27 (discussed in Sect. 3.3) have colors consistent with group C sources (Figs. 13 and 15): this is in agreement with our argument that they are accreting systems surrounded by a photo-ionized nebula or stellar wind, rather than SNRs. We also note that only 5 sources are clearly outliers, with much harder spectra than those of typical XRBs or SNRs. They could be analogous to the bright $\left(L_{\mathrm{x}} \sim 10^{38} \mathrm{erg} \mathrm{s}^{-1}\right)$ highmass XRBs SMC X-2 (Corbet et al. 2001) and LMC X-4 (La Barbera et al. 2001), whose X-ray spectra have powerlaw components with $\Gamma \lessgtr 1$.

XRBs in a soft and hard state are well separated in this kind of color-color diagrams. The number of bright sources detected in each state is comparable, suggesting that the bright XRBs in this galaxy are not preferentially found in either spectral state. It is less easy to separate BH from NS XRBs, or high-mass from low-mass XRBs, based on colors alone. As a comparison 

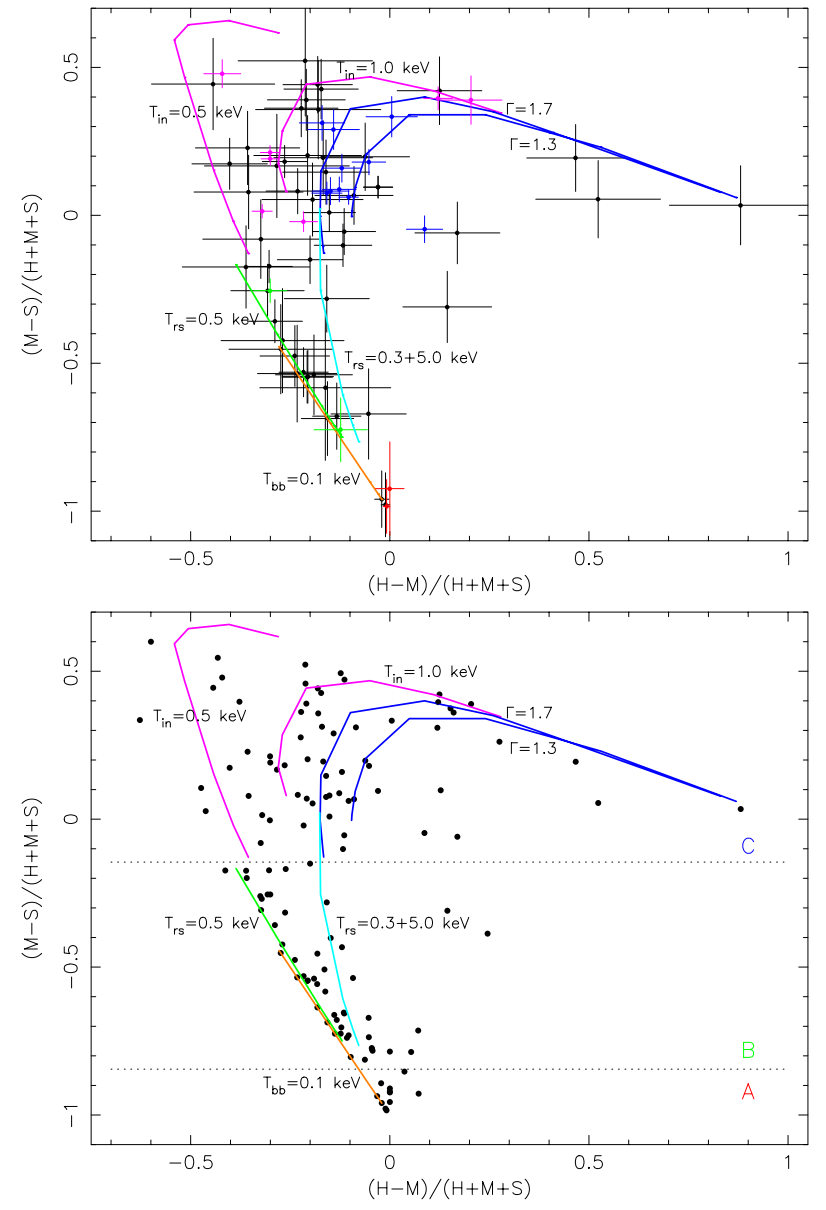

Fig. 15. Same as Fig. 14 for the alternative color indices. The three groups $(\mathrm{A}, \mathrm{B}, \mathrm{C})$ are separated by the horizontal dotted lines. (This figure is available in color in electronic form.)

with bright sources in the Galaxy and Magellanic Clouds, the high-mass XRBs LMC X-1 and LMC X-3 are almost always detected in a soft spectral state; on the other hand, Cyg X-1 is usually found in a hard state (with occasional transitions to a soft state), and most transient Be XRBs have a hard spectrum in outburst. BH XRBs with low-mass companions (e.g., GX339-4, XTE J1550-564) and NS low-mass XRBs (e.g., $4 \mathrm{U}$ 1728-34) also show transitions between soft and hard states. (See e.g., Lewin et al. 1995 for a review.) However, the spectral and color differences between the two "canonical" states are generally larger in BH XRBs than in NS XRBs (e.g., Sunyaev 2001; Sunyaev \& Revnivtsev 2000): BHs are harder when in the hard state, and softer when in the soft state. Hence, BH XRBs should have larger color excursions within the group $C$ sources. Repeated observations of the source colors over timescales of a few months/years may help distinguish NS from BH systems. Instead, we do not expect to see color transitions between different groups.

\subsection{Correlation with $\mathrm{H} \alpha$ emission}

The cumulative luminosity distributions of group A, B and $\mathrm{C}$ sources are not identical (Fig. 16). Group A (supersoft) and $\mathrm{B}$ (soft) sources have a much lower count rate: only $\approx 10 \%$ of them have $\gtrsim 100$ cts $\left(\approx 0.002 \mathrm{cts} \mathrm{s}^{-1}\right.$,

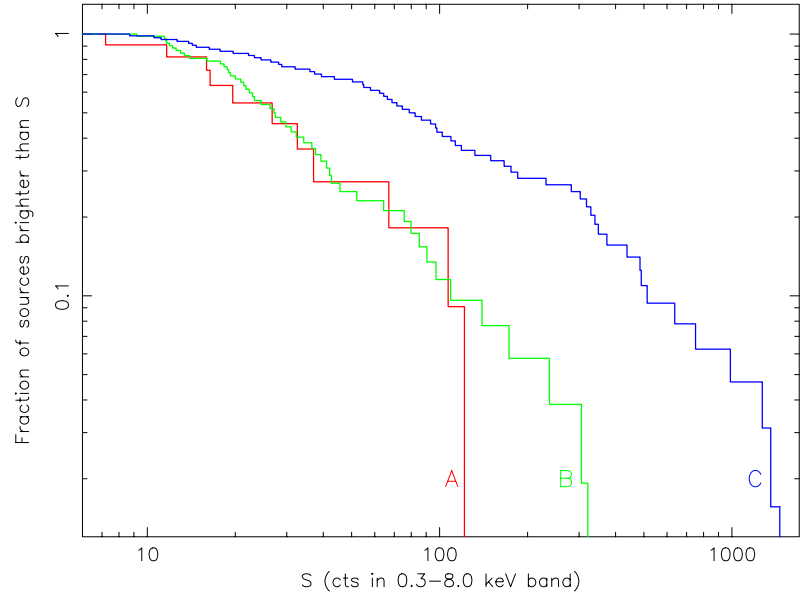

Fig. 16. Cumulative luminosity distributions of the three groups of sources (normalized to their total numbers) identified in Sect. 5.3. The distributions for groups A, B and C are plotted in red, green and blue respectively. (This figure is available in color in electronic form.)

corresponding to $\approx 3 \times 10^{37} \mathrm{erg} \mathrm{s}^{-1}$ ). The luminosity function of the group B sources is approximately a powerlaw with index -1 . Most of the bright sources belong to group $C: \approx 40 \%$ of the sources in this group have X-ray luminosities $\gtrsim 3 \times$ $10^{37} \mathrm{erg} \mathrm{s}^{-1}$. This fraction increases to $\approx 50 \%$ when we subtract the estimated background AGN contribution.

We investigated the spatial correlation between different groups of X-ray sources and the H II regions, which define the spiral arm structure and are indicators of young stellar populations. Overplotting the X-ray sources on a continuumsubtracted $\mathrm{H} \alpha$ map of M 83 (Fig. 17) shows that group B sources (green circles) are more closely associated with $\mathrm{H} \alpha$ emission (mostly found in the starburst nucleus and the spiral arms), than group A and C sources. We quantified this association by calculating the average $\mathrm{H} \alpha$ surface brightness within a circle of $5^{\prime \prime}$ radius around the position of each X-ray source, and plotting it as a function of the X-ray flux, for each group of sources (Fig. 18). The data do not show any correlation between X-ray count rates and $\mathrm{H} \alpha$ surface brightness for any of the three groups. The lack of faint X-ray sources associated with very bright $\mathrm{H} \alpha$ emission (near the bottom right of the plot) is due to the incompleteness of the X-ray sample: bright $\mathrm{H} \alpha$ emission is usually associated with strong diffuse X-ray emission (e.g., in the starburst nucleus), where faint $\mathrm{X}$-ray sources are more easily unresolved.

We then examined (Fig. 19) the cumulative distribution of the $\mathrm{H} \alpha$ surface brightness associated with the three groups of X-ray sources (the total number of sources is normalized to 1 for each group). This shows a significantly stronger $\mathrm{H} \alpha$ emission associated with group B sources: almost $80 \%$ of them are located in regions with $\mathrm{H} \alpha$ surface brightness $>2 \times 10^{35} \mathrm{erg} \mathrm{s}^{-1} \operatorname{arcsec}^{-2}$, compared to only $40 \%$ of group $\mathrm{C}$ sources. This is further evidence that group B sources come from a younger population. This difference is particularly evident in regions of intermediate $\mathrm{H} \alpha$ brightness, typical of $\mathrm{HII}$ regions in the spiral arms. For regions of very bright $\mathrm{H} \alpha$ emission $\left(\gtrsim 2 \times 10^{36} \mathrm{erg} \mathrm{s}^{-1} \operatorname{arcsec}^{-2}\right) \mathrm{B}$ and $\mathrm{C}$ sources have similar behavior: this corresponds to the starburst nucleus, 


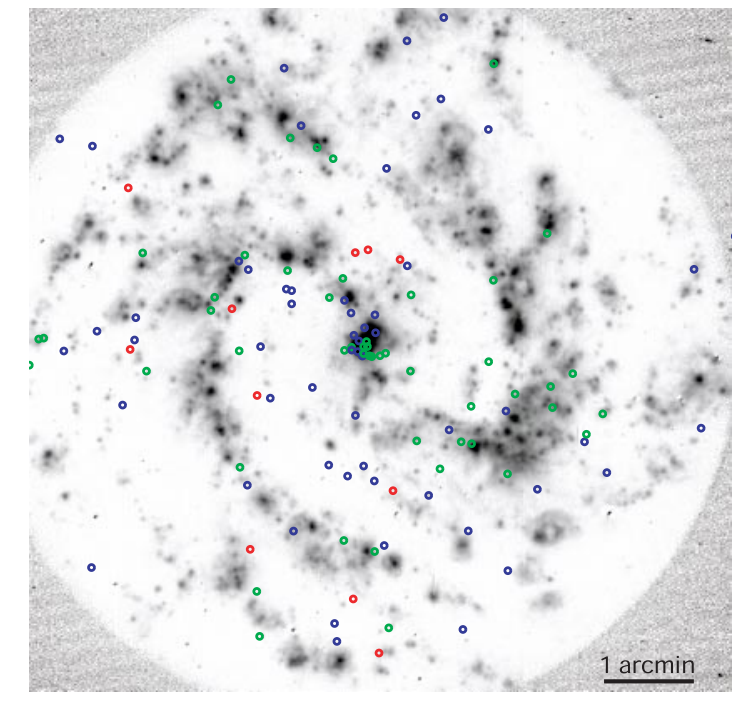

Fig. 17. Location of the discrete X-ray sources classified according to their color group (red for group A sources; green for group B; blue for group $\mathrm{C}$ ), overplotted onto a continuum-subtracted $\mathrm{H} \alpha$ image of M 83. North is up, East is left. The $\mathrm{H} \alpha$ image was taken by Stuart Ryder from the Anglo-Australian Telescope.

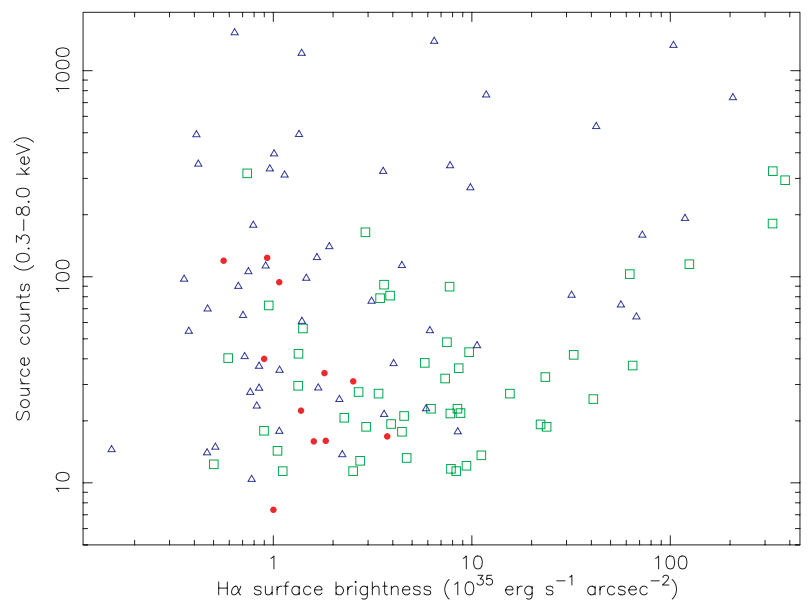

Fig. 18. X-ray source count rate versus the $\mathrm{H} \alpha$ surface brightness in the region around the source (average value in a circle of radius $5^{\prime \prime}$ ). Here red circles $=$ group A, green squares $=$ group B, blue triangles $=$ group $\mathrm{C}$ sources. The lack of detected sources near the bottom right corner of the diagram is due to the completeness limit of our X-ray sample in regions of high $\mathrm{H} \alpha$ emission: those regions are also characterized by strong, diffuse $\mathrm{X}$-ray emission, so that faint $\mathrm{X}$-ray sources cannot be detected.

where we expect to find a high concentration of both SNRs and high-mass XRBs.

Sources of group A (supersoft) are found only in regions of low $\mathrm{H} \alpha$ emission: this is consistent with an old population. However, it could also be due to a selection effect: supersoft sources embedded in a spiral arm or in the starburst nucleus (and hence associated with bright H II regions) are heavily absorbed and may not detected easily in the soft X-ray band. The small number of group A sources (11) also makes statistical errors larger.

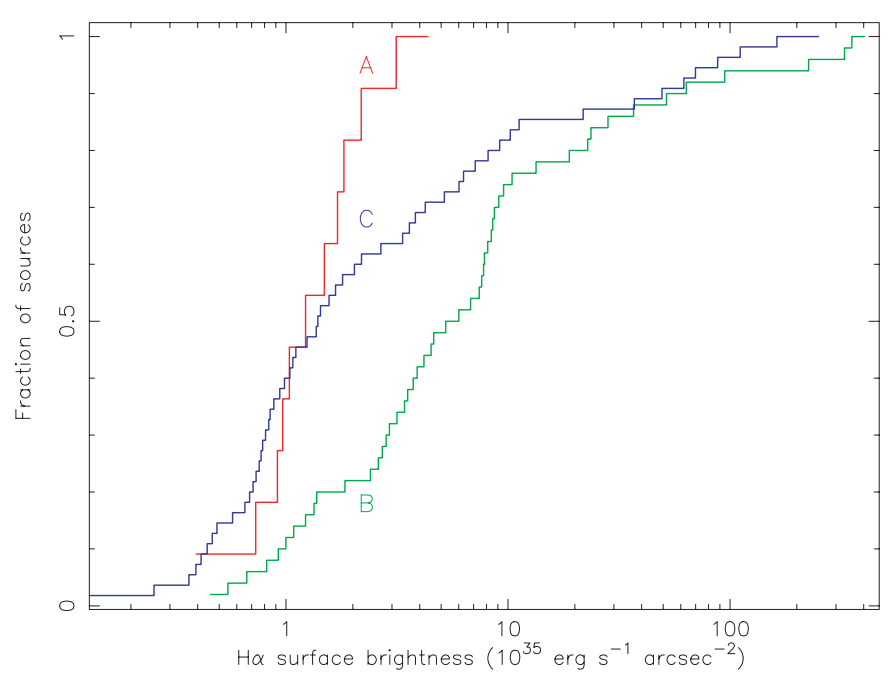

Fig. 19. Cumulative distributions of the $\mathrm{H} \alpha$ surface brightness associated to each group of X-ray sources.

\subsection{X-ray SNRs and other possible soft sources}

The previous results are consistent with the interpretation of the supersoft sources as old or intermediate-age nuclear-burning white dwarfs, and with the identification of the B sources as young SNRs (Prestwich et al. 2003). If this is the case, we expect their luminosity to remain constant over many years. Repeated deep observations of M 83 over the next few years will verify this conjecture.

We also looked for spatial correspondences between the X-ray sources and the optically-identified SNRs (Blair \& Long 2003, in preparation). The latter were identified with the $[\mathrm{S} \mathrm{II}] / \mathrm{H} \alpha$ criterion (Blair \& Long 1997), suitable to detect evolved remnants of all types over a lifetime of $\approx 20000$ $30000 \mathrm{yr}$ (e.g., Pannuti 2002 and references therein). Seven of the X-ray sources classified in group B are located within $5^{\prime \prime}$ of an optical SNR; two C sources and one A source are also within 5" of an SNR. However, most of the optical SNRs are not associated with any X-ray sources, and vice versa. Little overlapping between X-ray and optically-selected SNRs was also noticed in other nearby galaxies (Pannuti et al. 2002). Optical surveys tend to select a larger fraction of SNRs in low-density regions (hence, they tend to select type Ia events), while the brighter X-ray SNRs are those found in denser starforming regions. On the other hand, optical surveys based on the $[\mathrm{O} \mathrm{III}] / \mathrm{H} \alpha$ criterion would select mostly remnants from core-collapse events (type II, Ib,c) in young stellar populations. Correlations with X-ray sources would provide a more constraining test for their age and nature. This search is beyond the scope of this paper.

A detailed study of spatial correlations between X-ray and radio sources is also left to further work. A preliminary investigation shows that the continuum radio emission at $6.3 \mathrm{~cm}$ correlates well with the distribution of group B X-ray sources (Fig. 20). Diffuse thermal radio emission in spiral galaxies is usually associated with $\mathrm{H}$ II regions; diffuse non-thermal radio emission is thought to be produced by cosmic-ray electrons accelerated by SNRs. An association between diffuse radio 


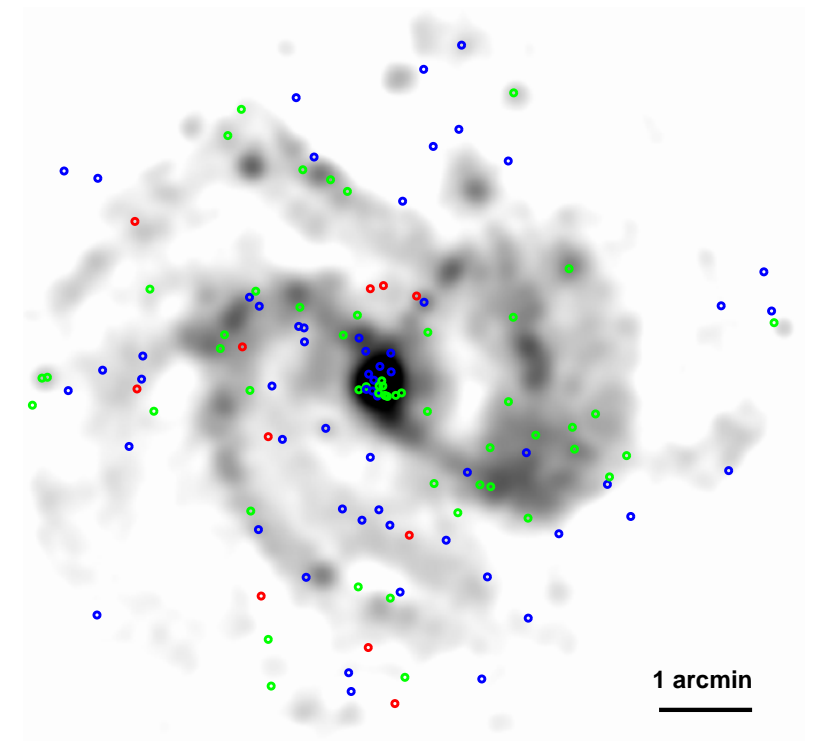

Fig. 20. Group A, B and C sources (green, red and blue circles respectively) overplotted on a map of the radio continuum emission at $6.3 \mathrm{~cm}$. The radio map is a combination of data from the VLA and Effelsberg radio telescopes, at a resolution of $12^{\prime \prime}$, and was produced by S. Sukumar, R. Allen, R. Beck \& N. Neininger. (This figure is available in color in electronic form.)

emission and group B X-ray sources would strengthen their interpretation as SNRs, or at least as a young population.

In addition to young SNRs, other classes of sources could in principle show X-ray colors consistent with our group B classification. For example, a blackbody or disk-blackbody spectrum with $k T \approx 0.1-0.2 \mathrm{keV}$ would have the correct X-ray colors. Standard accretion-disk models show that a $10-M_{\odot} \mathrm{BH}$ would have to be accreting at a rate $\lesssim 10^{-3} \dot{m}_{\text {Edd }}$ to have a temperature $k T \lesssim 0.2 \mathrm{keV}$ at the inner edge of the disk, if it extends to the innermost stable orbit of the Schwarzschild geometry. However, its luminosity would be $\lesssim 10^{36} \mathrm{erg} \mathrm{s}^{-1}$, too faint to be detected in our M 83 observation. Besides, typical BH candidates in the Galaxy tend to have a harder X-ray spectrum when detected at low accretion rates: their emission is then dominated by photons Compton-scattered in a hot corona, rather than direct emission from the accretion disk. Intermediate-mass BHs $\left(M \sim 10^{3}-10^{4} M_{\odot}\right)$ in a high state would have a thermal blackbody-like spectrum in the correct temperature range, but their luminosity would be $>10^{41} \mathrm{erg} \mathrm{s}^{-1}$, not observed in M 83 .

Transient X-ray pulsars with large photospheres may be a candidate for some of the soft sources. Some systems, such as RX J0059.2-7138 (Hughes 1994) are known to have a very soft $(k T \approx 40 \mathrm{eV})$ blackbody component in addition to their typical powerlaw component. The soft X-ray emission is produced by the Compton downscattering of harder X-rays emitted from near the surface of the compact object. With its emitted X-ray luminosity $\sim 10^{37} \mathrm{erg} \mathrm{s}^{-1}$, RX J0059.2-7138 would be a group B source in our classification scheme. Timevariability studies are required to distinguish this class of transient X-ray pulsars from X-ray SNRs. Finally, old low-mass neutron star XRBs may have an X-ray spectrum dominated by a soft thermal component at $k T \approx 100 \mathrm{eV}$, caused by the reprocessing of hard X-ray photons by the inner edge of an accretion disk. An example of this type of sources, which would also belong to group B, is the Galactic X-ray pulsar Her X-1 (e.g., Mavromatakis 1993).

\subsection{Soft sources in $M 83$ and $M 81$}

Another clue to determine the nature of the group B sources comes from a comparison of the X-ray colors of the discrete sources detected in other galaxies. We considered M 81, as it is another nearby spiral galaxy but it does not have a starburst nucleus like that of M 83. The foreground absorption in the direction of the two galaxies is very similar, hence this does not affect the hardness and color comparison. In order to compare our results with those of Swartz et al. (2002, 2003), we used the Chandra ACIS count rates for the M 83 sources obtained by $D$. Swartz with the same extraction routines and in the same energy bands. Hence, the soft band is defined here as the 0.2$1.0 \mathrm{keV}$ channel energy range, instead of the $0.3-1.0 \mathrm{keV}$ band used in the rest of this paper. Medium and hard bands are defined as before.

The color-color plots (Fig. 21) and the cumulative hardness distributions (Fig. 22) show similar populations of supersoft sources and XRBs for the two galaxies. However, M 81 has fewer group B sources. This difference may be caused either by physical properties (different star-formation activity and history of the two galaxies) or by the higher inclination angle of the M 81 disk. If this group of young soft sources are concentrated in the spiral arms, they may be more highly absorbed and less likely to be detected. However, the fact that there is no difference in the relative detection of the other groups of sources suggests that the former explanation is more likely. As discussed in Sect. 5.3, a significant fraction of group B sources in M 83 is located in the starburst nuclear region (10 out of 50) and in other young star-forming regions where the interstellar gas density is higher. It is natural that fewer of these sources are in the bulge of M 81, which is characterized by an older stellar population and little interstellar gas.

\section{Summary}

We have identified 127 discrete sources in a 51-ks Chandra ACIS-S3 observation of the starburst spiral galaxy M 83, with a detection limit of $\approx 3 \times 10^{36} \mathrm{cts} \mathrm{s}^{-1}$ in the $0.3-8.0 \mathrm{keV}$ band. Most of the bright sources have spectra typical of XRBs, either in a soft state (thermal component at $\sim 1 \mathrm{keV}$ plus powerlaw with $\Gamma \approx 2.5$ ), or in a hard state (powerlaw with $\Gamma \approx 1.5$ ). Two bright sources show emission lines on a hard powerlaw continuum, and are probably XRBs surrounded by a photoionized nebula or stellar wind, though the alternative that they may be very young SNRs cannot be ruled out. Among the other bright sources, we also modelled the spectra of two SNR candidates, with optically-thin thermal plasma emission at temperatures $\sim 0.5 \mathrm{keV}$ and emission lines from $\mathrm{Mg}$ and $\mathrm{Si}$. The two brightest supersoft sources have blackbody temperatures $k T \approx 60 \mathrm{eV}$ and luminosities $\sim 10^{38} \mathrm{erg} \mathrm{s}^{-1}$. Two candidate $\mathrm{X}$-ray pulsars are detected with periods $\approx 200 \mathrm{~s}$.

An analysis of the X-ray colors, luminosity and spatial distribution suggests that the discrete source population can be 

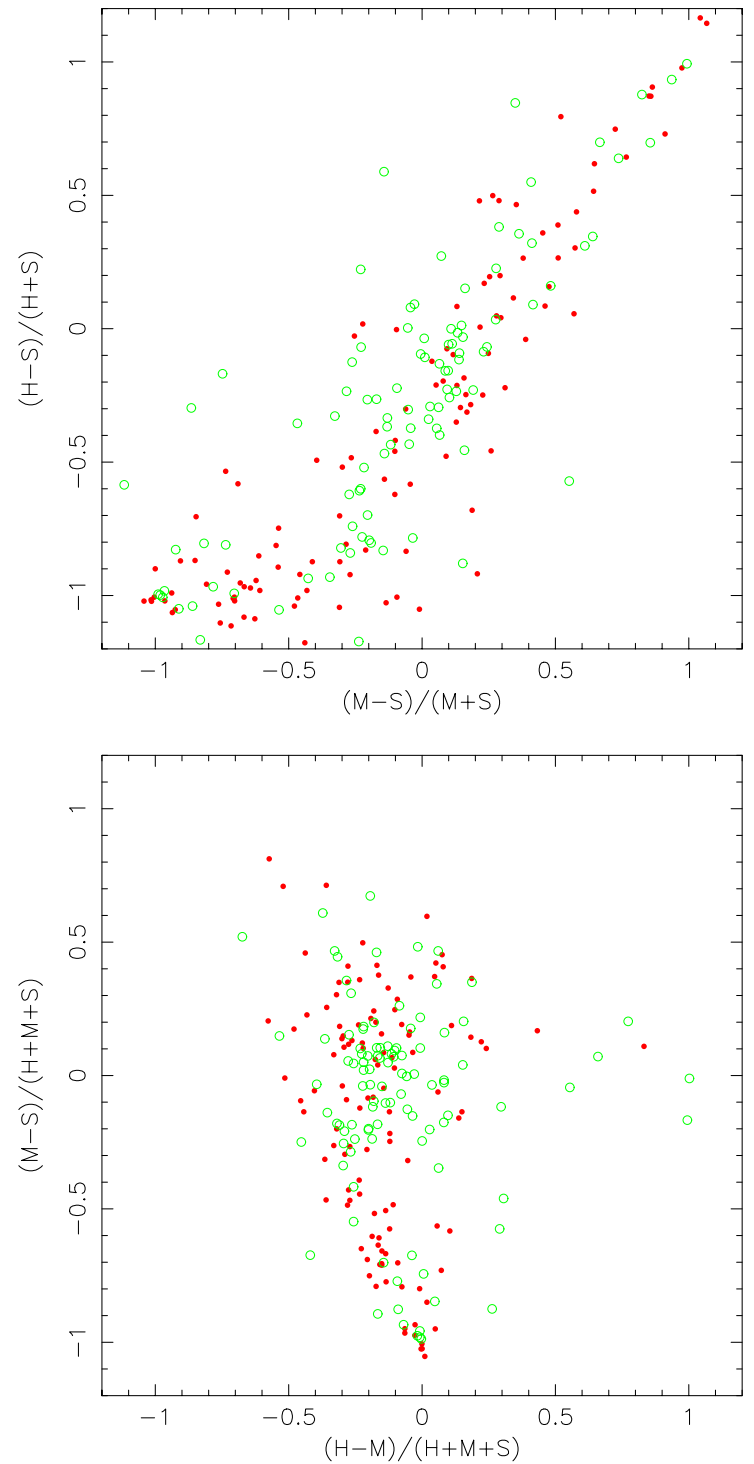

Fig. 21. Color-color plots for X-ray sources in M 83 and M 81, for conventional and alternative color indices (as defined in Sect. 5.2). Here the soft band is defined as $0.2-1.0 \mathrm{keV}$. Filled red circles represent M 83 sources, and open green circles represent M 81 sources. Error bars have been omitted. (This figure is available in color in electronic form.)

divided into three distinct groups. The first group $(\approx 10 \%$ of the detected sources) comprises "classical" supersoft sources with blackbody-like spectra at temperatures $<100 \mathrm{eV}$. They do not correlate strongly with $\mathrm{H} \alpha$ emission, an indicator of recent star formation, suggesting that they belong to an old or intermediate-age stellar population. This is consistent with their interpretation as nuclear-burning white dwarfs. The second group $(\approx 40 \%$ of the total) consists of soft sources with little or no detected emission above $2 \mathrm{keV}$. The brightest sources in this group have unabsorbed luminosities $\approx 10^{38} \mathrm{erg} \mathrm{s}^{-1}$, but for most of the others we estimate luminosities $\sim 10^{37} \mathrm{erg} \mathrm{s}^{-1}$. They are mostly found in regions with high $\mathrm{H} \alpha$ emission (H II regions in the spiral arms and the starburst nucleus). Most of them are probably core-collapse SNRs, although other physical systems may occasionally show similarly soft X-ray colors (for example, accreting neutron stars in high-mass XRBs with large
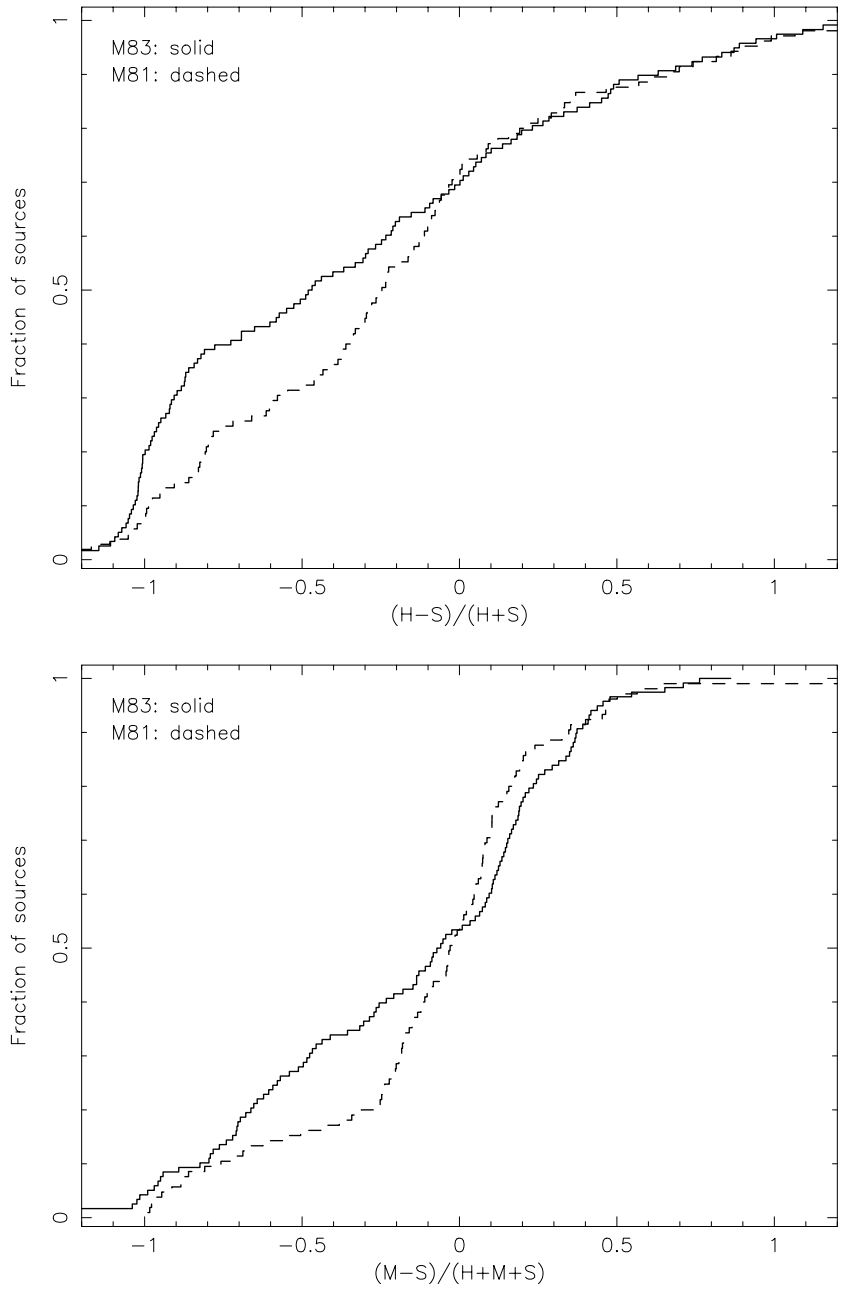

Fig. 22. The cumulative color distribution of the sources in M 83 (solid line) and M 81 (dashed line). $\mathrm{S}$ is defined here as the $0.2-1.0 \mathrm{keV}$ band.

photospheres). A comparison with the source colors in the spiral galaxy M 81 (where star formation is currently less active) confirms that the fraction of sources detected in this group is related to the recent level of star formation. The sources in the third group are mostly XRBs, reaching higher X-ray luminosities than sources in the other two groups. Being a mixture of old low-mass and young high-mass XRBs, the whole group appears to be of intermediate age when correlated with the $\mathrm{H} \alpha$ emission. The color-color diagrams allow us to distinguish between sources in a soft and hard state, and to disentangle the effects of absorption versus intrinsic spectral hardness.

Acknowledgements. We thank Rosanne Di Stefano, Stefan Immler, Phil Kaaret, Roy Kilgard, Albert Kong, Manfred Pakull, Andrea Prestwich, Doug Swartz, Andreas Zezas for valuable discussions. We thank Doug Swartz for providing us his 3-band Chandra ACIS count rates for sources in M 81 and M 83, so that we could carry out a statistical comparison of two populations with an identical data-reduction procedure. We also thank: Darragh O'Donoghue for the use of his EAGLE Fourier transform code; Rainer Beck and Stuart Ryder for the radio and $\mathrm{H} \alpha$ images respectively; the Aspen Center for Physics, where part of this work was carried out, for the support and hospitality. We are grateful to the referee (Wolfgang Pietsch) for useful suggestions that made this paper much clearer. 


\section{Appendix A: Discrete sources in the Chandra ACIS S3 image of M 83}

Table A.1. M 83 sources detected at the 3- $\sigma$ level in the $0.3-8.0 \mathrm{keV}$ band, and respective net fluxes in a soft, medium and hard band. Counts in a given band are given in brackets when the source was detected at less than 3- $\sigma$ level in that band. The exposure time was $50.851 \mathrm{ks}$. The ROSAT cross-identification refers to the HRI sources listed by Immler et al. (1999); SW02 is the cross-identification with Soria \& Wu (2002).

\begin{tabular}{|c|c|c|c|c|c|c|c|c|c|}
\hline No. & CXOU Name & RA (2000) & Dec (2000) & $F_{0.3-8}(\mathrm{cts})$ & $F_{0.3-1}(\mathrm{cts})$ & $F_{1-2}(\mathrm{cts})$ & $F_{2-8}(\mathrm{cts})$ & $R O S A T$ & SW02 \\
\hline 1 & J133640.8-295118 & 133640.89 & -295118.2 & $23.5 \pm 08.5$ & $18.8 \pm 05.3$ & $(3.0 \pm 2.0)$ & $(0.0 \pm 0.0)$ & & \\
\hline 2 & J133641.0-295110 & 33641.01 & -295110.7 & $67.7 \pm 10.2$ & $22.8 \pm 07.3$ & $40.1 \pm 07.4$ & $(13.0 \pm 5.2)$ & & 1 \\
\hline 3 & J133641.4-295045 & 133641.41 & -295045.1 & $294.0 \pm 19.8$ & $49.7 \pm 08.2$ & $126.7 \pm 12.8$ & $89.2 \pm 11.2$ & & 2 \\
\hline 4 & J133643.1-295254 & 133643.16 & -295254.3 & $10.4 \pm 03.7$ & $(4.0 \pm 2.0)$ & $(5.0 \pm 3.0)$ & $(0.5 \pm 0.5)$ & & \\
\hline 5 & J133643.5-295107 & 133643.53 & -295107.2 & $324.7 \pm 20.3$ & $29.7 \pm 06.1$ & $120.0 \pm 12.2$ & $121.3 \pm 12.5$ & H08 & 3 \\
\hline 6 & J133648.0-295324 & 133648.05 & -295324.2 & $60.8 \pm 08.9$ & $13.2 \pm 04.2$ & $23.2 \pm 05.1$ & $13.0 \pm 04.0$ & & 4 \\
\hline 7 & J133648.2-295244 & 133648.27 & -295244.8 & $19.3 \pm 05.0$ & $10.4 \pm 03.6$ & $(6.0 \pm 3.0)$ & $(0.5 \pm 0.5)$ & & \\
\hline 8 & J133649.1-295258 & 133649.11 & -295258.5 & $317.5 \pm 18.8$ & $189.8 \pm 14.5$ & $109.7 \pm 11.0$ & $15.2 \pm 04.2$ & $\mathrm{H} 12 \mathrm{a}$ & 5 \\
\hline 9 & J133649.2-295303 & 133649.22 & -295303.5 & $112.9 \pm 11.4$ & $35.9 \pm 06.6$ & $53.7 \pm 07.7$ & $12.6 \pm 03.9$ & $\mathrm{H} 12 \mathrm{~b}$ & 6 \\
\hline 10 & J133649.8-295217 & 133649.82 & -295217.7 & & $28.2 \pm 06.1$ & & $(0.5 \pm 0.5)$ & & 7 \\
\hline 11 & J133650.8-295240 & 133650.87 & -295240.5 & $27.1 \pm 06.4$ & $20.6 \pm 05.4$ & $(4.0 \pm 2.0)$ & $(0.5 \pm 0.5)$ & & \\
\hline 12 & J133650.9-295226 & 133650.97 & -295226.3 & $21.8 \pm 05.8$ & $14.7 \pm 04.7$ & $(4.0 \pm 2.0)$ & $(0.5 \pm 0.5)$ & & \\
\hline 13 & J133651.1-295043 & 133651.15 & -295043.2 & $19.2 \pm 05.4$ & $10.6 \pm 03.9$ & $(6.0 \pm 3.0)$ & $(0.5 \pm 0.5)$ & & \\
\hline 14 & J133651.6-295335 & 13365 & 335.5 & $124.0 \pm 12.0$ & $42.6 \pm 07.1$ & $52.4 \pm 07.5$ & $24.7 \pm 05.2$ & H14 & 8 \\
\hline 15 & J133652.8-295231 & 133652.80 & -295231.4 & $32.1 \pm 06.7$ & $20.6 \pm 05.4$ & $10.8 \pm 03.5$ & $(0.5 \pm 0.5)$ & & \\
\hline 16 & J133653.1-295430 & 133653.19 & -295430.4 & $27.5 \pm 06.0$ & $(5.0 \pm 2.0)$ & $15.2 \pm 04.0$ & $(5.5 \pm 2.5)$ & & \\
\hline 17 & J133653.1-295325 & 133653.19 & -295325.3 & $43.1 \pm 07.7$ & $34.5 \pm 06.6$ & $(5.5 \pm 3.2)$ & $(3.2 \pm 2.5)$ & & 9 \\
\hline 18 & J133653.2-295242 & 133653.27 & -295242.9 & & $19.1 \pm 05.3$ & $16.0 \pm 04.2$ & $(3.5 \pm 2$ & & 10 \\
\hline 19 & J133653.6-295600 & 13365 & -295600.9 & $30.0 \pm 0$ & & & & & \\
\hline 20 & J133653.9-294848 & 133653.92 & -294848.8 & $32.6 \pm 06.9$ & $28.1 \pm 06.2$ & $(4.0 \pm 2.0)$ & $(0.5 \pm 0.5)$ & & 11 \\
\hline 21 & J133653.9-295114 & 133653.93 & -295114.7 & $89.5 \pm 10.2$ & $43.7 \pm 07.1$ & $30.6 \pm 05.7$ & $13.1 \pm 03.9$ & & 12 \\
\hline 22 & J133654.1-295209 & 133654.17 & -295209.6 & $12.3 \pm 04.1$ & $10.5 \pm 03.5$ & $(2.0 \pm 1.0)$ & $(0.5 \pm 0.5)$ & & \\
\hline 23 & J133654.1-294933 & 133654.18 & -294933.1 & & $(12.6 \pm 5.6)$ & $(12.5 \pm 5.2)$ & & & \\
\hline 24 & J133655.0-295304 & 5.06 & -295304.9 & $25.5 \pm 0$ & $15.9 \pm 04.6$ & $11.2 \pm 03.7$ & $(0.0 \pm 0.0)$ & & \\
\hline 25 & J133655.0-295239 & 133655.09 & -295239.6 & $29.6 \pm 06.2$ & $24.2 \pm 05.4$ & $(4.0 \pm 2.0)$ & $(0.5 \pm 0.5)$ & & 13 \\
\hline 26 & J133655.2-295403 & 133655.23 & -295403.6 & $98.4 \pm 10.5$ & $41.7 \pm 07.1$ & $36.1 \pm 06.1$ & $24.4 \pm 05.1$ & & 14 \\
\hline 27 & J133655.5-295510 & 33655.50 & -295510.1 & $352.3 \pm 19.2$ & $94.7 \pm 10.1$ & $151.3 \pm 12.5$ & $108.6 \pm 10.7$ & H15 & 15 \\
\hline 28 & J133655.6-295303 & 5.60 & -295303.6 & \pm 07.9 & $23.5 \pm 06.1$ & $8.7 \pm 03.2$ & $15.6 \pm 04.1$ & & 16 \\
\hline 29 & J133656.2-295255 & 133656.22 & -295255.5 & $22.9 \pm 05.5$ & $(5.5 \pm 2.3)$ & $12.3 \pm 03.7$ & $(6.8 \pm 2.6)$ & & 17 \\
\hline 30 & J133656.5-294817 & 133656.51 & -294817.7 & & $(2.0 \pm 1.0)$ & $11.3 \pm 03.5$ & $(7.0 \pm 3.0)$ & & \\
\hline 31 & J133656.6-294912 & 133656.65 & -294912.6 & $311.6 \pm 18.8$ & $53.3 \pm 07.7$ & $145.0 \pm 12.7$ & $95.1 \pm 10.5$ & & 18 \\
\hline 32 & J133656.6-295321 & 133656.69 & -295321.8 & $14.3 \pm 04.5$ & $(11.0 \pm 4.0)$ & $(1.0 \pm 1.0)$ & $(2.0 \pm 1.0)$ & & \\
\hline 33 & J133657.2-295339 & 133657.28 & -295339.7 & $490.6 \pm 22.7$ & $162.8 \pm 13.3$ & $201.6 \pm 14.4$ & $126.8 \pm 11.4$ & & 19 \\
\hline 34 & J133657.5-294729 & 133657.52 & -294729.2 & $96.4 \pm 12.5$ & $35.1 \pm 08.1$ & $42.5 \pm 07.6$ & $32.6 \pm 07.6$ & & 20 \\
\hline 35 & J133657.8-295303 & 133657.89 & -295303.0 & $81.0 \pm 09.4$ & $61.7 \pm 08.1$ & $18.6 \pm 04.5$ & $(0.5 \pm 0.5)$ & & 21 \\
\hline 36 & J133657.9-294923 & 1336 & -294923.6 & $35.2 \pm 06.7$ & $(1.0 \pm 1.0)$ & $12.3 \pm 03.7$ & 16.9 & & 22 \\
\hline 37 & J133658.2-295124 & 133658.20 & -295124.6 & $20.7 \pm 05.3$ & $(15.5 \pm 6.2)$ & $10.6 \pm 03.6$ & $(3.0 \pm 2.0)$ & & 23 \\
\hline 38 & J133658.2-295216 & 133658.23 & -295216.0 & $164.3 \pm 13.4$ & $91.9 \pm 10.0$ & $63.0 \pm 08.1$ & $12.3 \pm 03.7$ & & 24 \\
\hline${ }^{a} 39$ & J133658.3-295105 & 133658.39 & -295105.1 & $124.9 \pm 11.8$ & $(2.0 \pm 1.0)$ & $54.1 \pm 07.6$ & $70.4 \pm 08.6$ & & 26 \\
\hline 40 & J133658.4-294833 & 133658.41 & -294833.4 & $76.1 \pm 10.2$ & $26.1 \pm 07.7$ & $21.2 \pm 05.1$ & $35.2 \pm 06.9$ & & 25 \\
\hline 41 & J133658.7-295100 & 133658.76 & -295100.9 & $16.0 \pm 04.8$ & $17.9 \pm 05.1$ & $(0.5 \pm 0.5)$ & $(0.5 \pm 0.5)$ & & \\
\hline 42 & J133659.1-295336 & 133659.13 & -295336.5 & $34.1 \pm 06.5$ & $32.8 \pm 06.2$ & $(0.5 \pm 0.5)$ & $(0.5 \pm 0.5)$ & & 27 \\
\hline 43 & J133659.3-295508 & 133659.35 & -295508.9 & $11.4 \pm 03.9$ & $8.0 \pm 03.2$ & $(1.0 \pm 1.0)$ & $(0.5 \pm 0.5)$ & & \\
\hline 44 & J133659.4-294959 & 133659.46 & -294959.3 & $1214.1 \pm 36.0$ & $399.2 \pm 20.7$ & $474.2 \pm 22.4$ & $347.9 \pm 19.3$ & H17 & 28 \\
\hline 45 & J133659.5-295203 & 133659.52 & -295203.9 & $36.0 \pm 12.5$ & $29.7 \pm 10.0$ & $(9.0 \pm 3.5)$ & $(0.0 \pm 0.0)$ & $\left({ }^{b}\right)$ & 29 \\
\hline 46 & J133659.5-295413 & 133659.59 & -295413.5 & $23.6 \pm 05.6$ & $(1.0 \pm 1.0)$ & $11.8 \pm 03.6$ & $9.1 \pm 03.2$ & & \\
\hline 47 & J133659.8-295205 & 133659.81 & -295205.5 & $270.4 \pm 21.0$ & $85.7 \pm 13.9$ & $137.6 \pm 12.7$ & $62.2 \pm 08.2$ & $\left({ }^{b}\right)$ & 30 \\
\hline 48 & J133659.8-295525 & 133659.85 & -295525.9 & $31.1 \pm 06.2$ & $24.2 \pm 05.1$ & $(1.0 \pm 1.0)$ & $(2.0 \pm 1.0)$ & & \\
\hline 49 & J133700.0-295150 & 133700.03 & -295150.2 & $192.1 \pm 22.7$ & $77.9 \pm 15.5$ & $79.8 \pm 10.9$ & $48.5 \pm 07.4$ & $\left({ }^{b}\right)$ & 31 \\
\hline 50 & J133700.0-295138 & 133700.06 & -295138.1 & $17.7 \pm 05.8$ & $(6.0 \pm 3.0)$ & $12.3 \pm 04.1$ & $(0.5 \pm 0.5)$ & & \\
\hline 51 & $\mathrm{~J} 133700.0-295417$ & 133700.08 & -295417.5 & $11.7 \pm 04.0$ & $9.7 \pm 03.6$ & $(1.0 \pm 1.0)$ & $(0.5 \pm 0.5)$ & & \\
\hline 52 & J133700.1-295330 & 133700.11 & -295330.0 & $36.8 \pm 06.4$ & $(2.0 \pm 1.0)$ & $20.7 \pm 04.6$ & $13.1 \pm 03.7$ & & 32 \\
\hline 53 & J133700.2-295206 & 133700.23 & -295206.3 & $41.8 \pm 11.6$ & $28.1 \pm 10.4$ & $19.5 \pm 6.3$ & $(1.7 \pm 1.7)$ & $\left({ }^{b}\right)$ & 33 \\
\hline
\end{tabular}

\footnotetext{
${ }^{a}$ Background radio galaxy.
} 
Table A.1. continued.

\begin{tabular}{|c|c|c|c|c|c|c|c|c|c|}
\hline No. & CXOU Name & RA (2000) & Dec (2000) & $F_{0.3-8}$ (cts) & $F_{0.3-1}$ (cts) & $F_{1-2}(\mathrm{cts})$ & $F_{2-8}(\mathrm{cts})$ & $R O S A T$ & SW0 \\
\hline 54 & J133700.3-295205 & 133700.36 & -295205.6 & $37.1 \pm 12.1$ & $24.9 \pm 09.2$ & $(6.2 \pm 4.9)$ & $(0.5 \pm 0.5)$ & $\left({ }^{b}\right)$ & \\
\hline 55 & J133700.4-295054 & 33700.42 & -295054.3 & $94.1 \pm 10.4$ & $91.2 \pm 10.1$ & & $(0.0 \pm 0.0)$ & & \\
\hline 56 & J133700.4-295159 & 133700.45 & -295159.5 & $325.4 \pm 37.8$ & $290.4 \pm 33.5$ & $45.0 \pm 21.9$ & $(3.2 \pm 3.2)$ & $\left({ }^{b}\right)$ & \\
\hline 57 & J133700.5-295156 & 133700.51 & -295156.1 & $294.0 \pm 39.7$ & $220.9 \pm 31.5$ & $77.0 \pm 24.2$ & $(4.8 \pm 4.8)$ & $\left({ }^{b}\right)$ & \\
\hline 58 & J133700.6-295146 & 133700.60 & -295146.7 & $63.9 \pm 13.0$ & $(8.1 \pm 4.9)$ & $26.8 \pm 06.6$ & $17.4 \pm 04.6$ & $\left(b^{b}\right)$ & \\
\hline 59 & J133700.6-295159 & 133700.65 & -295159.5 & $181.1 \pm 39.0$ & $112.6 \pm 35.0$ & $45.0 \pm 14.7$ & $(2.0 \pm 2.0)$ & $\left({ }^{b}\right)$ & \\
\hline 60 & J133700.6-295319 & 133700.65 & -295319.9 & $395.0 \pm 20.2$ & $125.6 \pm 11.5$ & $160.4 \pm 12.8$ & $110.2 \pm 10.6$ & & \\
\hline 61 & J133700.6-295204 & 133700.66 & -295204.0 & $115.1 \pm 32.4$ & $85.7 \pm 21.0$ & $(32.3 \pm 13.7)$ & $(0.5 \pm 0.5)$ & $\left({ }^{b}\right)$ & \\
\hline 62 & J133700.7-295205 & 133700.72 & -295205.9 & $159.3 \pm 30.5$ & $(18.2 \pm 8.8)$ & $99.2 \pm 13.0$ & $66.0 \pm 08.7$ & $\left({ }^{b}\right)$ & \\
\hline 63 & J133700.9-295155 & 133700.90 & -295155.7 & $739.4 \pm 36.6$ & $204.6 \pm 24.8$ & $274.4 \pm 20.9$ & $252.9 \pm 16.6$ & $(b, c)$ & \\
\hline 64 & J133700.9-295202 & 133700.97 & -295202.8 & $1327.1 \pm 45.7$ & $570.9 \pm 33.4$ & $589.5 \pm 27.2$ & $164.8 \pm 13.4$ & $\left({ }^{b}\right)$ & \\
\hline 65 & J133701.0-295245 & 133701.07 & -295245.8 & $113.4 \pm 11.2$ & $51.3 \pm 07.6$ & $39.5 \pm 06.5$ & $25.8 \pm 05.2$ & $\mathrm{H} 21$ & \\
\hline 66 & J133701.0-295056 & 133701.08 & -295056.2 & $22.4 \pm 05.9$ & $21.8 \pm 05.5$ & $(1.0 \pm 1.0)$ & $(0.5 \pm 0.5)$ & & \\
\hline 67 & J133701.1-295151 & 13370 & -295151.8 & $81.4 \pm 15.4$ & $\pm 9.1)$ & $47.5 \pm 09.0$ & $14.0 \pm$ & $\left({ }^{b}\right)$ & \\
\hline 68 & J133701.1-295449 & 133701.18 & -295449.5 & $123.5 \pm 11.4$ & $123.4 \pm 11.4$ & $(0.5 \pm 0.5)$ & $(0.0 \pm 0.0)$ & & \\
\hline 69 & J133701.2-295201 & 133701.27 & -295201.7 & $73.0 \pm 11.7$ & $(9.0 \pm 7.5)$ & $(13.0 \pm 5.4)$ & $51.4 \pm 07.9$ & $\left({ }^{b}\right)$ & \\
\hline 70 & J133701.2-295159 & 133701.28 & -295159.9 & $103.0 \pm 25.2$ & $69.2 \pm 10.0$ & $40.1 \pm 09.4$ & $(5.0 \pm 2.0)$ & $\left(b^{b}\right)$ & \\
\hline 71 & J133701.3-295136 & 133701.31 & -295136.9 & & & $(3.0 \pm 2.0)$ & $54.8 \pm 07.6$ & & \\
\hline 72 & J133701.4-295326 & 133701.49 & -295326.7 & $488.9 \pm 22.5$ & $75.1 \pm 08.8$ & $307.9 \pm 17.7$ & $103.3 \pm 10.4$ & $\mathrm{H} 20$ & \\
\hline 73 & J133701.6-295128 & 133701.64 & -295128.4 & $535.7 \pm 23.9$ & $224.7 \pm 15.6$ & $213.3 \pm 14.9$ & $97.4 \pm 10.1$ & $\mathrm{H} 23$ & \\
\hline 74 & J133701.6-295201 & 133701.65 & -295201.9 & $22.9 \pm 06.8$ & $21.6 \pm 06.4$ & $(2.0 \pm 1.0)$ & $(0.5 \pm 0.5)$ & $\left({ }^{b}\right)$ & \\
\hline 75 & J133701.6-295410 & 133701.68 & -295410 & & & $(2.0 \pm 1$ & & & \\
\hline 76 & J133701.7-294743 & 133701.72 & -294743.0 & & & $71.7 \pm 09.5$ & $48.5 \pm 07.9$ & & \\
\hline 77 & J133701.7-295113 & 133701.72 & -295113.5 & $27.1 \pm 06.1$ & $20.6 \pm 05.1$ & $(3.0 \pm 2.0)$ & & & \\
\hline 78 & J133702.0-295518 & 133702.03 & -295518.0 & $333.8 \pm 18.6$ & $112.2 \pm 10.9$ & $96.5 \pm 09.9$ & $125.7 \pm 11.4$ & $\mathrm{H} 22$ & \\
\hline 79 & J133702.1-295505 & 133702.16 & -295505.9 & $54.4 \pm 07.9$ & $17.0 \pm 04.5$ & $19.5 \pm 04.6$ & $10.4 \pm 03.5$ & & \\
\hline 80 & J133702.2-294952 & 133702.23 & -294952.9 & & & & & & \\
\hline 81 & J133702.4-295126 & 133 & -295 & & & & & & \\
\hline 82 & J133702.4-295319 & 133702.47 & -29 5319.4 & $97.4 \pm 10.1$ & $13.9 \pm 03.9$ & $51.9 \pm 07.3$ & $31.5 \pm$ & & \\
\hline 83 & J133703.0-294945 & 133703.06 & -294945.4 & $18.7 \pm 05.2$ & $(10.6 \pm 6.2)$ & & & & \\
\hline 84 & J133703.3-295226 & 133703.31 & & & & & & & \\
\hline 85 & $\mathrm{~J} 133703.8$ & 1337 & -294 & $346.0=$ & 114. & $141.7 \pm$ & & & \\
\hline 86 & J133704.2-295403 & 133704.29 & -295403.8 & $1389.2 \pm 37.8$ & $406.8 \pm 20.4$ & $702.4 \pm 26.8$ & $283.4 \pm 17.0$ & $\mathrm{H} 26$ & \\
\hline 87 & J133704.3-295130 & 133704.38 & -295130.7 & $41.0 \pm 07.0$ & $16.2 \pm 04.5$ & $19.4 \pm 04.6$ & $(5.0 \pm 2.0)$ & & \\
\hline 88 & J133704.3-295121 & 133704.38 & -295121.7 & $1527.8 \pm 39.7$ & $469.6 \pm 22.0$ & $762.6 \pm 27.9$ & $302.0 \pm 17.6$ & $\mathrm{H} 27 \mathrm{a}$ & \\
\hline 89 & $\mathrm{~J} 1337$ & 4.46 & -294938.8 & $12.1 \pm 04.1$ & $7.8 \pm 03.3$ & $(2.0 \pm 1.0)$ & $(1.0 \pm 1.0)$ & & \\
\hline 90 & J133704.5-295108 & 133704.59 & -295 & $2 \pm 04.4$ & & & & & \\
\hline 91 & J133704.6-295120 & 133704.66 & -29 5120.6 & $69.8 \pm 09.0$ & $20.6 \pm 05.0$ & $31.0 \pm 06.0$ & $19.6 \pm 04.7$ & $\mathrm{H} 27 \mathrm{~b}$ & \\
\hline 92 & J133704.7-294851 & 133704.77 & -294851.9 & $37.9 \pm 07.0$ & $(10.6 \pm 4.5)$ & $16.2 \pm 04.2$ & & & \\
\hline 93 & J133705.4-295234 & 133705.48 & & & & $39.8 \pm 06.4$ & $51.3 \pm$ & & \\
\hline 94 & J133706.0-295159 & 133706.00 & -295159.5 & $17.8 \pm 04.6$ & $(1.0 \pm 1.0)$ & $9.3 \pm 03.2$ & $7.3 \pm 02.8$ & & \\
\hline 95 & J133706.0-295514 & 133706.04 & -295514.7 & $72.6 \pm 08.9$ & $53.2 \pm 07.5$ & $15.3 \pm 04.0$ & $(1.0 \pm 1.0)$ & & \\
\hline 96 & J133706.1-295232 & 133706.18 & -295232.4 & $119.8 \pm 11.5$ & $110.9 \pm 10.9$ & & & & \\
\hline 97 & J133706.1-295444 & 133706.19 & -295444.3 & $42.3 \pm 06.9$ & $36.1 \pm 06.3$ & $6.7 \pm 02.6$ & $(0.5=$ & & \\
\hline 98 & J133706.5-295416 & 133706.54 & -295416.0 & $15.9 \pm 04.5$ & $15.3 \pm 04.2$ & $(0.5 \pm 0.5)$ & $(0.0 \pm 0.0)$ & & \\
\hline 99 & J133706.6-295107 & 133706.63 & -295107.6 & $13.7 \pm 04.4$ & $(3.0 \pm 2.0)$ & $4.3 \pm 02.2$ & $6.0 \pm 02.0$ & & \\
\hline 100 & J133706.6-295332 & 133706.67 & -295332.8 & $25.4 \pm 05.7$ & $8.2 \pm 03.2$ & $9.8 \pm 03.3$ & $(5.0 \pm 2.0)$ & & \\
\hline 101 & J133706.8-295057 & 133706.81 & -295057.8 & $17.7 \pm 05.6$ & $(8.3 \pm 5.8)$ & $(2.0 \pm 1.0)$ & & & \\
\hline 102 & J133707.0-295320 & 133707.06 & -295320.8 & $11.4 \pm 04.1$ & $(10.0 \pm 4.0)$ & $(1.0 \pm 1.0)$ & $(0.5 \pm 0.5)$ & & \\
\hline 103 & J133707.1-295202 & 133707.10 & -295202.3 & $17.9 \pm 04.7$ & $11.5 \pm 03.7$ & $(4.0 \pm 2.0)$ & $(1.0 \pm 1.0)$ & & \\
\hline 104 & J133707.1-295101 & 133707.12 & -295101.8 & $762.4 \pm 28.2$ & $22.5 \pm 05.4$ & $325.7 \pm 18.3$ & $418.8 \pm 20.8$ & & \\
\hline 105 & J133707.4-295133 & 133707.47 & -295133.9 & $16.8 \pm 04.8$ & $15.7 \pm 04.5$ & $(0.5 \pm 0.5)$ & $(0.5 \pm 0.5)$ & & \\
\hline 106 & J133707.5-294859 & 133707.53 & -294859.5 & & & & & & \\
\hline 107 & J133708.2-294916 & 133708.21 & -294916.6 & $13.6 \pm 04.5$ & $(12.0 \pm 5.0)$ & $(1.0 \pm 1.0)$ & $(1.0 \pm 1.0)$ & & \\
\hline 108 & J133708.3-295126 & 133708.37 & -295126.2 & $21.7 \pm 05.7$ & $13.3 \pm 04.5$ & $(4.0 \pm 2.0)$ & $(1.0 \pm 1.0)$ & & \\
\hline 109 & J133708.5-295135 & 133708.57 & -295135.2 & $11.4 \pm 04.1$ & $9.2 \pm 03.5$ & $(1.0 \pm 1.0)$ & $(0.0 \pm 0.0)$ & & \\
\hline 110 & J133711.9-295215 & 133711.90 & -295215.8 & $56.1 \pm 08.1$ & $45.5 \pm 07.1$ & $8.3 \pm 03.0$ & $(0.5 \pm 0.5)$ & & 7 \\
\hline
\end{tabular}

\footnotetext{
${ }^{b}$ Contributing to the nuclear source $\mathrm{H} 19$, unresolved by ROSAT
}

${ }^{c} \mathrm{X}$-ray source coincident with the IR/optical/UV nucleus of the galaxy. 
Table A.1. continued.

\begin{tabular}{|c|c|c|c|c|c|c|c|c|c|}
\hline No. & CXOU Name & RA (2000) & Dec (2000) & $F_{0.3-8}(\mathrm{cts})$ & $F_{0.3-1}(\mathrm{cts})$ & $F_{1-2}(\mathrm{cts})$ & $F_{2-8}(\mathrm{cts})$ & $R O S A T$ & SW02 \\
\hline 111 & J133712.1-295056 & 133712.10 & -295056.4 & $18.7 \pm 05.3$ & $15.8 \pm 04.7$ & $(1.0 \pm 1.0)$ & $(2.0 \pm 1.0)$ & & \\
\hline 112 & J133712.4-295140 & 3712.46 & -295140.0 & & & & & & 73 \\
\hline 113 & J13371 & 3712.52 & -295155.0 & 177.9 & & $69.2 \pm$ & $104.9 \pm$ & & 74 \\
\hline 114 & $\mathrm{~J} 133712$. & & & & & & & & \\
\hline 115 & J133712.8-295012 & 133712.84 & -295012.4 & & $37.3 \pm 06.4$ & & & & 75 \\
\hline 116 & J133713 & 13371 & -29523 & & & & & & \\
\hline 117 & J13371 & & -29514 & & & $56.3 \pm$ & 32.6 & & 76 \\
\hline 118 & J13371 & & -29 & & & $24.7=$ & 15.6 & & 77 \\
\hline 119 & & & -29 & & & & & & \\
\hline 120 & J133716.1-295202 & 133716.18 & -295202.4 & $14.9 \pm 04.1$ & $(1.0 \pm 1.0)$ & $4.9 \pm 02.2$ & & & \\
\hline 121 & J133716.3-294939 & & -294939.5 & $480.6 \pm 23.0$ & $112.1 \pm 11.0$ & $199.5 \pm 14.9$ & $174.0 \pm 13.9$ & & 78 \\
\hline 122 & J13371 & & -29 & $91.5 \pm 10.1$ & $61.6 \pm 08.2$ & $28.6 \pm 05.6$ & $(2.0$ & & 79 \\
\hline 123 & J133717.4-295154 & 133717.48 & -295154.2 & $21.1 \pm 07.2$ & $(12.0 \pm 5.0)$ & $(6.0 \pm 3.0)$ & $(1.0 \pm 1.0)$ & & \\
\hline 124 & J133717.9-295211 & 133717.97 & -295211.9 & $40.3 \pm 06.6$ & $21.4 \pm 04.8$ & $10.9 \pm 03.3$ & $(5.0 \pm 2.0)$ & & 80 \\
\hline 125 & J133718.8-295014 & & -29 5014.4 & & & & & & \\
\hline 126 & J133719.6-295131 & & & & & $20.0 \pm 04.6$ & $58.5 \pm 07.9$ & & 81 \\
\hline 127 & J133722.1-295207 & 133722.13 & -295207.8 & $11.6 \pm 03.7$ & $(1.0 \pm 1.0)$ & $5.8 \pm 02.4$ & $(2.0 \pm 1.0)$ & & \\
\hline
\end{tabular}

\section{Appendix B: Fit parameters of selected bright sources}

Table B.1. XSPEC best-fit parameters for the galactic nucleus (No. 63 in Table A.1). Here and hereafter, the Galactic line-of-sight column density has been fixed at $4.0 \times 10^{20} \mathrm{~cm}^{-2}$.

\begin{tabular}{lc}
\hline \hline model: $w^{2}{ }^{2} s_{\text {Gal }} \times$ wabs $\times$ powerlaw \\
\hline$n_{\mathrm{H}}\left(\times 10^{21} \mathrm{~cm}^{-2}\right)$ & $1.25_{-0.93}^{+0.80}$ \\
$\Gamma$ & $1.45_{-0.24}^{+0.16}$ \\
$K_{\mathrm{pl}}\left(\times 10^{-5}\right)$ & $1.9_{-0.5}^{+0.3}$ \\
\hline$\chi_{v}^{2}($ d.o.f. $)$ & $1.06(49)$ \\
$L_{0.3-8}\left(\times 10^{38} \mathrm{erg} \mathrm{s}^{-1}\right)$ & $2.3_{-0.1}^{+0.2}$ \\
\hline
\end{tabular}

Table B.2. XSPEC best-fit parameters for the two brightest supersoft sources. (An asterisk indicates that we could not determine an error for that fit parameter.)

\begin{tabular}{lrr}
\hline \hline parameter & No. 68 & No. 96 \\
\hline \hline \multicolumn{1}{c}{ model: wabs $_{\text {Gal }} \times$ wabs $\times$ blackbody } \\
\hline$n_{\mathrm{H}}\left(\times 10^{21} \mathrm{~cm}^{-2}\right)$ & $1.4_{-0.5}^{+1.4}$ & $4.7_{-1.0}^{+2.3}$ \\
$T_{\mathrm{bb}}(\mathrm{keV})$ & $0.065_{-0.013}^{+0.014}$ & $0.058_{-0.023}^{+0.032}$ \\
$K_{\mathrm{bb}}\left(\times 10^{-6}\right)$ & $2.3_{-0.4}^{+0.3}$ & $48_{-25}^{+35}$ \\
\hline$\chi_{v}^{2}($ d.o.f. $)$ & $1.01(7)$ & $0.51(5)$ \\
$L_{0.3-8}\left(\times 10^{38} \mathrm{erg} \mathrm{s}^{-1}\right)$ & $0.9_{-0.4}^{+3.4}$ & {$[>1]$} \\
\hline \hline
\end{tabular}

model: wabs $_{\mathrm{Gal}} \times$ wabs $\times$ raymond-smith

\begin{tabular}{lrr}
\hline$n_{\mathrm{H}}\left(\times 10^{21} \mathrm{~cm}^{-2}\right)$ & $1.9_{-1.2}^{+1.5}$ & $4.5_{-4.5}^{+150}$ \\
$T_{\mathrm{rs}}(\mathrm{keV})$ & $0.087_{-0.027}^{+0.033}$ & $0.082_{-0.013}^{+0.007}$ \\
$\mathrm{Z}($ metal ab. $)$ & $0.01_{-0.01}^{+0.04}$ & $0.02_{-0.02}^{+0.14}$ \\
$K_{\mathrm{rs}}\left(\times 10^{-3}\right)$ & $7.7_{-4.5}^{+8.0}$ & $59_{-*}^{+*}$ \\
\hline$\chi_{v}^{2}($ d.o.f. $)$ & $1.08(6)$ & $0.41(4)$ \\
$L_{0.3-8}\left(\times 10^{38} \mathrm{erg} \mathrm{s}^{-1}\right)$ & $2.0_{-1.5}^{+10.2}$ & {$[>1]$} \\
\hline
\end{tabular}


Table B.3. XSPEC best-fit parameters for emission-line sources. (Parameters listed as "undetermined" are those for which we could not obtain meaningful values with XSPEC.)

\begin{tabular}{|c|c|c|c|c|}
\hline parameter & No. 3 & No. 8 & No. 27 & No. 56 \\
\hline \multicolumn{5}{|c|}{ model: wabs $_{\text {Gal }} \times$ wabs $\times$ powerlaw } \\
\hline$n_{\mathrm{H}}\left(\times 10^{21} \mathrm{~cm}^{-2}\right)$ & $1.3_{-0.9}^{+0.8}$ & $2.2_{-0.6}^{+0.9}$ & $0.7_{-0.7}^{+0.6}$ & $>104$ \\
\hline$\Gamma$ & $1.51_{-0.16}^{+0.30}$ & $3.64_{-0.35}^{+0.54}$ & $1.35_{-0.15}^{+0.14}$ & $>10$ \\
\hline$K_{\mathrm{pl}}\left(\times 10^{-6}\right)$ & $10.3_{-2.8}^{+4.0}$ & $14.0_{-4.1}^{+6.6}$ & $8.1_{-2.0}^{+2.6}$ & [undeterm] \\
\hline$\chi_{v}^{2}$ (d.o.f.) & $1.11(30)$ & $1.22(25)$ & $1.06(30)$ & [undeterm] \\
\hline$L_{0.3-8}\left(\times 10^{38} \mathrm{erg} \mathrm{s}^{-1}\right)$ & $1.2_{-0.1}^{+0.1}$ & $1.6_{-0.5}^{+1.8}$ & $1.1_{-0.1}^{+0.1}$ & [undeterm] \\
\hline \multicolumn{5}{|c|}{ model: wabs $_{\text {Gal }} \times$ wabs $\times$ bremsstrahlung } \\
\hline$n_{\mathrm{H}}\left(\times 10^{21} \mathrm{~cm}^{-2}\right)$ & $1.1_{-0.9}^{+1.0}$ & $0.8_{-0.6}^{+0.7}$ & $0.6_{-0.6}^{+0.7}$ & $9.7_{-0.8}^{+0.7}$ \\
\hline$T_{\text {br }}(\mathrm{keV})$ & $10.8_{-5.5}^{+17.5}$ & $0.71_{-0.19}^{+0.0}$ & $27.7_{-18.6}^{+* .0}$ & $0.10_{-0.03}^{+0.04}$ \\
\hline$K_{\mathrm{br}}\left(\times 10^{-5}\right)$ & $1.4_{-0.2}^{-0.5}$ & $2.8_{-0.4}^{+0.5}$ & $\begin{array}{r}-18.6 \\
1.4_{-0.3}^{+1.0}\end{array}$ & $1.0_{-0.3}^{+0.4} 10^{-0.03}$ \\
\hline$\chi_{v}^{2}$ (d.o.f.) & $1.05(30)$ & $1.23(25)$ & $1.05(30)$ & $1.55(24)$ \\
\hline$L_{0.3-8}\left(\times 10^{38} \mathrm{erg} \mathrm{s}^{-1}\right)$ & $1.1_{-0.1}^{+0.1}$ & $0.6_{-0.2}^{+0.2}$ & $1.1_{-0.1}^{+0.1}$ & [undeterm] \\
\hline \multicolumn{5}{|c|}{ model: wabs $_{\text {Gal }} \times$ wabs $\times$ raymond-smith } \\
\hline$n_{\mathrm{H}}\left(\times 10^{21} \mathrm{~cm}^{-2}\right)$ & $0.9_{-0.7}^{+1.0}$ & $0.9_{-0.9}^{+0.5}$ & $0.7_{-0.7}^{+0.6}$ & $<0.5$ \\
\hline$T_{\mathrm{rs}}(\mathrm{keV})$ & $12.1_{-5.3}^{+32.9}$ & $0.65_{-0.13}^{+0.40}$ & $24.6_{-16.5}^{+39.4}$ & $0.67_{-0.08}^{+0.10}$ \\
\hline $\mathrm{Z}$ (metal ab.) & 1 [fixed] & $<0.1$ & 1 [fixed] & $<0.2$ \\
\hline$K_{\mathrm{rS}}\left(\times 10^{-5}\right)$ & $2.6_{-*}^{+2.2}$ & $9.6_{-*}^{+2.8}$ & $3.4_{-*}^{+2.1}$ & $2.9_{-0.8}^{+2.1}$ \\
\hline$\chi_{v}^{2}$ (d.o.f.) & $1.04(30)$ & $1.28(24)$ & $1.05(30)$ & $1.27(23)$ \\
\hline$L_{0.3-8}\left(\times 10^{38} \mathrm{erg} \mathrm{s}^{-1}\right)$ & $1.2_{-0.2}^{+0.2}$ & $0.6_{-0.2}^{+0.2}$ & $1.1_{-0.2}^{+0.2}$ & $0.4_{-0.2}^{+0.2}$ \\
\hline \multicolumn{5}{|c|}{ model: wabs $_{\text {Gal }} \times$ wabs $\times($ powerlaw $/$ bremss continuum + Gaussians $)$} \\
\hline$n_{\mathrm{H}}\left(\times 10^{21} \mathrm{~cm}^{-2}\right)$ & $0.2_{-0.2}^{+0.8}$ & $0.6_{-0.5}^{+0.5}$ & $0.5_{-0.4}^{+0.6}$ & $9.6_{-0.6}^{+0.6}$ \\
\hline$\Gamma$ & $1.29_{-0.20}^{+0.29}$ & & $1.30_{-0.18}^{+0.45}$ & \\
\hline$K_{\mathrm{pl}}\left(\times 10^{-6}\right)$ & $7.0_{-2.5}^{+3.28}$ & & $7.1_{-0.9}^{+0.18}$ & \\
\hline$T_{\mathrm{br}}(\mathrm{keV})$ & & $0.72_{-0.20}^{+0.16}$ & & $0.15_{-0.05}^{+0.05}$ \\
\hline$K_{\mathrm{br}}\left(\times 10^{-5}\right)$ & & $2.5_{-1.1}^{+1.9}$ & & $526_{-60}^{+60}$ \\
\hline \multirow[t]{2}{*}{ line 1} & $E=1.32_{-0.05}^{+0.04} \mathrm{keV}$ & $E=1.27_{-0.03}^{+0.03} \mathrm{keV}$ & $E=1.33_{-0.08}^{+0.08}$ & \multirow{3}{*}{$\begin{array}{r}E=1.50_{-0.05}^{+0.04} \mathrm{keV} \\
\text { [undeterm] }\end{array}$} \\
\hline & $E W=85_{-60}^{+108} \mathrm{eV}$ & & $E W=73_{-73}^{+94} \mathrm{eV}$ & \\
\hline line 2 & $\begin{array}{c}E=1.51_{-0.03}^{+0.04} \mathrm{keV} \\
E W=135_{-88}^{+89} \mathrm{eV}\end{array}$ & & & \\
\hline \multirow[t]{2}{*}{ line 3} & $E=1.85_{-0.03}^{+0.04} \mathrm{keV}$ & & $E=1.91_{-0.04}^{+0.04} \mathrm{keV}$ & \multirow{4}{*}{$\begin{array}{r}E=1.89_{-0.07}^{+0.03} \mathrm{keV} \\
\quad \text { [undeterm] }\end{array}$} \\
\hline & $E W=154_{-131}^{+98} \mathrm{eV}$ & & $E W=189_{-111}^{-114} \mathrm{eV}$ & \\
\hline \multirow[t]{2}{*}{ line 4} & $E=2.60_{-0.10}^{+0.19} \mathrm{keV}$ & & & \\
\hline & $E W=293_{-204}^{+293} \mathrm{eV}$ & & & \\
\hline$\chi_{v}^{2}$ (d.o.f.) & $0.82(22)$ & 0.88 & $0.85(24)$ & $1.01(22)$ \\
\hline$L_{0.3-8}\left(\times 10^{38} \mathrm{erg} \mathrm{s}^{-1}\right)$ & $1.1_{-0.1}^{+0.1}$ & $0.5_{-0.1}^{+0.2}$ & $1.1_{-0.1}^{+0.1}$ & [undeterm] \\
\hline
\end{tabular}


Table B.4. XSPEC best-fit parameters for other selected bright sources in the ACIS-S3 chip. (An asterisk indicates that we could not obtain a meaningful best-fit value for that parameter.)

\begin{tabular}{|c|c|c|c|c|c|c|c|c|}
\hline parameter & No. 5 & No. 31 & No. 33 & [No. 39] & No. 44 & No. 60 & No. 64 & No. 72 \\
\hline \multicolumn{9}{|c|}{ model: wabs $_{\mathrm{Gal}} \times$ wabs $\times$ powerlaw } \\
\hline $\begin{array}{l}n_{\mathrm{H}}\left(\times 10^{21} \mathrm{~cm}^{-2}\right) \\
\Gamma \\
K_{\mathrm{pl}}\left(\times 10^{-5}\right) \\
\end{array}$ & $\begin{array}{r}3.5_{-1.3}^{+2.0} \\
1.62_{-0.33}^{+0.33} \\
1.6_{-0.6}^{+0.6}\end{array}$ & $\begin{array}{l}1.05_{-0.94}^{+0.84} \\
1.39_{-0.32}^{+0.21} \\
0.85_{-0.25}^{+0.36}\end{array}$ & $\begin{array}{r}1.15_{-0.51}^{+0.47} \\
1.68_{-0.17}^{+0.16} \\
1.3_{-0.3}^{+0.3}\end{array}$ & $\begin{array}{r}7.7_{-3.1}^{+7.5} \\
1.43_{-0.41}^{+0.51} \\
0.59_{-0.09}^{+0.10}\end{array}$ & $\begin{array}{r}0.74_{-0.29}^{+0.26} \\
1.40_{-0.10}^{+0.12} \\
2.7_{-0.3}^{+0.4}\end{array}$ & $\begin{array}{r}1.13_{-0.77}^{+0.48} \\
1.58_{-0.24}^{+0.22} \\
1.0_{-0.2}^{+0.2}\end{array}$ & $\begin{array}{r}2.1_{-0.4}^{+0.4} \\
2.77_{-0.18}^{+0.21} \\
6.3_{-0.9}^{+1.0}\end{array}$ & $\begin{array}{r}8.0_{-1.4}^{+1.7} \\
3.13_{-0.11}^{+0.57} \\
7.4_{-2.1}^{+3.3}\end{array}$ \\
\hline $\begin{array}{l}\chi_{v}^{2} \text { (d.o.f.) } \\
L_{0.3-8}\left(\times 10^{38} \mathrm{erg} \mathrm{s}^{-1}\right)\end{array}$ & $\begin{array}{r}0.88(33) \\
1.9_{-0.4}^{+0.2}\end{array}$ & $\begin{array}{r}0.88(27) \\
1.1_{-0.1}^{+0.1}\end{array}$ & $\begin{array}{r}1.08(41) \\
1.3_{-0.1}^{+0.1}\end{array}$ & $\begin{array}{r}0.72(9) \\
\text { [Radio gal.] }\end{array}$ & $\begin{array}{r}1.24(67) \\
3.6_{-0.1}^{+0.1}\end{array}$ & $\begin{array}{r}0.60(34) \\
1.1_{-0.1}^{+0.1}\end{array}$ & $\begin{array}{r}0.94(94) \\
5.0_{-0.8}^{+1.1}\end{array}$ & $\begin{array}{r}1.58(27) \\
7.1_{-2.5}^{+4.8}\end{array}$ \\
\hline \multicolumn{9}{|c|}{ model: wabs $_{\mathrm{Gal}} \times$ wabs $\times$ disk-blackbody } \\
\hline $\begin{array}{l}n_{\mathrm{H}}\left(\times 10^{21} \mathrm{~cm}^{-2}\right) \\
T_{\text {in }}(\mathrm{keV}) \\
K_{\mathrm{dbb}}\left(\times 10^{-2}\right) \\
\end{array}$ & & & & & & & $\begin{array}{r}0.13_{-0.13}^{+0.22} \\
0.68_{-0.07}^{+0.07} \\
2.9_{-1.0}^{+1.7} \\
\end{array}$ & $\begin{array}{r}3.4_{-1.0}^{+1.1} \\
0.72_{-0.09}^{+0.09} \\
1.6_{-0.7}^{+1.3} \\
\end{array}$ \\
\hline $\begin{array}{l}\chi_{v}^{2} \text { (d.o.f.) } \\
L_{0.3-8}\left(\times 10^{38} \mathrm{erg} \mathrm{s}^{-1}\right)\end{array}$ & & & & & & & $\begin{array}{r}1.13(94) \\
2.0_{-0.1}^{+0.1}\end{array}$ & $\begin{array}{r}1.21(27) \\
1.4_{-0.1}^{+0.3}\end{array}$ \\
\hline \multicolumn{9}{|c|}{ model: wabs $_{\text {Gal }} \times$ wabs $\times$ bmc } \\
\hline $\begin{array}{l}n_{\mathrm{H}}\left(\times 10^{21} \mathrm{~cm}^{-2}\right) \\
T_{\mathrm{bb}}(\mathrm{keV}) \\
\Gamma \\
K_{\mathrm{bmc}}\left(\times 10^{-7}\right)\end{array}$ & & & & & & & $\begin{array}{r}0.65_{-0.64}^{+0.61} \\
0.18_{-0.10}^{+0.06} \\
2.56_{-0.23}^{+0.19} \\
11.9_{-0.4}^{+0.4}\end{array}$ & $\begin{array}{r}1.1_{-0.9}^{+1.5} \\
0.53_{-0.12}^{+0.04} \\
6.3_{-*}^{+*} \\
7.1_{-0.6}^{+0.8}\end{array}$ \\
\hline $\begin{array}{l}\chi_{v}^{2} \text { (d.o.f.) } \\
L_{0.3-8}\left(\times 10^{38} \mathrm{erg} \mathrm{s}^{-1}\right)\end{array}$ & & & & & & & $\begin{array}{r}0.90(92) \\
2.5_{-0.3}^{+0.6}\end{array}$ & $\begin{array}{r}1.28(25) \\
1.0_{-0.1}^{+0.1}\end{array}$ \\
\hline
\end{tabular}

Table B.4. continued.

\begin{tabular}{|c|c|c|c|c|c|c|c|c|}
\hline parameter & No. 73 & No. 78 & No. 85 & No. 86 & No. 88 & No. 104 & No. 113 & No. 121 \\
\hline \multicolumn{9}{|c|}{ model: wabs $_{\text {Gal }} \times$ wabs $\times$ powerlaw } \\
\hline $\begin{array}{l}n_{\mathrm{H}}\left(\times 10^{21} \mathrm{~cm}^{-2}\right) \\
\Gamma \\
K_{\mathrm{pl}}\left(\times 10^{-5}\right)\end{array}$ & $\begin{array}{r}1.8_{-0.6}^{+0.6} \\
2.30_{-0.19}^{+0.26} \\
2.0_{-0.4}^{+0.6}\end{array}$ & $\begin{array}{r}<0.14 \\
0.98_{-0.18}^{+0.20} \\
0.51_{-0.07}^{+0.06}\end{array}$ & $\begin{array}{l}0.84_{-0.57}^{+0.54} \\
1.65_{-0.23}^{+0.27} \\
0.85_{-0.20}^{+0.23}\end{array}$ & $\begin{array}{r}3.9_{-0.4}^{+0.5} \\
2.69_{-0.15}^{+0.15} \\
9.2_{-1.2}^{+1.3}\end{array}$ & $\begin{array}{r}2.9_{-0.4}^{+0.4} \\
2.37_{-0.14}^{+0.08} \\
7.5_{-0.9}^{+1.1}\end{array}$ & $\begin{array}{r}14.6_{-2.5}^{+4.5} \\
2.12_{-0.27}^{+0.32} \\
9.3_{-3.2}^{+5.2}\end{array}$ & $\begin{array}{r}16.7_{-6.8}^{+8.6} \\
2.17_{-0.44}^{+0.74} \\
2.4_{-0.3}^{+0.4}\end{array}$ & $\begin{array}{r}1.17_{-0.85}^{+0.79} \\
1.29_{-0.17}^{+0.15} \\
1.2_{-0.2}^{+0.4}\end{array}$ \\
\hline$\overline{\chi_{v}^{2} \text { (d.o.f.) }}$ & $1.09(41)$ & $1.17(29)$ & $0.86(30)$ & $0.75(105)$ & $0.80(105)$ & $0.95(63)$ & $0.53(14)$ & $0.79(46)$ \\
\hline$L_{0.3-8}\left(\times 10^{38} \mathrm{erg} \mathrm{s}^{-1}\right)$ & $1.6_{-0.3}^{+0.2}$ & $1.0_{-0.1}^{+0.1}$ & $0.9_{-0.1}^{+0.1}$ & $7.2_{-0.9}^{+1.4}$ & $5.9_{-0.7}^{+0.8}$ & $7.7_{-1.5}^{+3.5}$ & $2.0_{-0.8}^{+2.8}$ & $1.8_{-0.1}^{+0.1}$ \\
\hline \multicolumn{9}{|c|}{ model: wabs $_{\text {Gal }} \times$ wabs $\times$ disk-blackbody } \\
\hline $\begin{array}{l}n_{\mathrm{H}}\left(\times 10^{21} \mathrm{~cm}^{-2}\right) \\
T_{\text {in }}(\mathrm{keV}) \\
K_{\text {dbb }}\left(\times 10^{-2}\right)\end{array}$ & $\begin{array}{r}<0.39 \\
0.94_{-0.16}^{+0.15} \\
0.33_{-0.13}^{+0.46}\end{array}$ & & & $\begin{array}{r}1.32_{-0.26}^{+0.29} \\
0.86_{-0.06}^{+0.07} \\
1.7_{-0.5}^{+0.6}\end{array}$ & $\begin{array}{r}0.90_{-0.23}^{+0.24} \\
0.91_{-0.07}^{+0.08} \\
1.4_{-0.4}^{+0.5}\end{array}$ & $\begin{array}{r}8.9_{-2.1}^{+2.4} \\
1.46_{-0.21}^{+0.27} \\
0.27_{-0.09}^{+0.12}\end{array}$ & $\begin{array}{r}10.2_{-3.3}^{+5.8} \\
1.50_{-0.44}^{+0.69} \\
0.06_{-*}^{+0.21}\end{array}$ & \\
\hline $\begin{array}{l}\chi_{v}^{2} \text { (d.o.f.) } \\
L_{0.3-8}\left(\times 10^{38} \mathrm{erg} \mathrm{s}^{-1}\right)\end{array}$ & $\begin{array}{r}1.42(41) \\
0.9_{-0.1}^{+0.1}\end{array}$ & & & $\begin{array}{r}0.68(105) \\
3.0_{-0.1}^{+0.1}\end{array}$ & $\begin{array}{r}0.81(112) \\
3.0_{-0.1}^{+0.1}\end{array}$ & $\begin{array}{r}0.87(63) \\
4.0_{-0.3}^{+0.3}\end{array}$ & $\begin{array}{r}0.54(14) \\
1.0_{-0.1}^{+0.2}\end{array}$ & \\
\hline \multicolumn{9}{|c|}{ model: wabs $_{\text {Gal }} \times$ wabs $\times$ bmc } \\
\hline $\begin{array}{l}n_{\mathrm{H}}\left(\times 10^{21} \mathrm{~cm}^{-2}\right) \\
T_{\mathrm{bb}}(\mathrm{keV}) \\
\Gamma \\
K_{\mathrm{bmc}}\left(\times 10^{-7}\right)\end{array}$ & $\begin{array}{r}14.9_{-1.4}^{+19.7} \\
0.19_{-0.07}^{+0.08} \\
1.67_{-0.58}^{+0.54} \\
6.7_{-0.3}^{+0.3}\end{array}$ & & & $\begin{array}{r}0.8_{-0.4}^{+0.3} \\
0.36_{-0.04}^{+0.07} \\
3.25_{-0.34}^{+0.29} \\
14.6_{-0.8}^{+5.3}\end{array}$ & $\begin{array}{r}0.55_{-0.20}^{+0.24} \\
0.31_{-0.02}^{+0.07} \\
2.61_{-0.21}^{+0.28} \\
14.3_{-0.7}^{+1.2}\end{array}$ & $\begin{array}{r}5.1_{-2.9}^{+3.6} \\
0.76_{-0.31}^{+0.19} \\
1.3_{-0.2}^{+2.7} \\
3.0_{-0.6}^{+0.6}\end{array}$ & $\begin{array}{r}9.1_{-3.3}^{+17.5} \\
0.60_{-*}^{+0.36} \\
* \\
7.6_{-*}^{+*}\end{array}$ & \\
\hline $\begin{array}{l}\chi_{v}^{2} \text { (d.o.f.) } \\
L_{0.3-8}\left(\times 10^{38} \mathrm{erg} \mathrm{s}^{-1}\right)\end{array}$ & $\begin{array}{r}0.93(39) \\
1.4_{-0.3}^{+1.5}\end{array}$ & & & $\begin{array}{r}0.72(103) \\
2.7_{-0.1}^{+0.1}\end{array}$ & $\begin{array}{r}0.79(110) \\
3.1_{-0.1}^{+0.1}\end{array}$ & $\begin{array}{r}0.89(61) \\
3.3_{-0.4}^{+0.4}\end{array}$ & $\begin{array}{r}0.54(12) \\
0.9_{-*}^{+*}\end{array}$ & \\
\hline
\end{tabular}



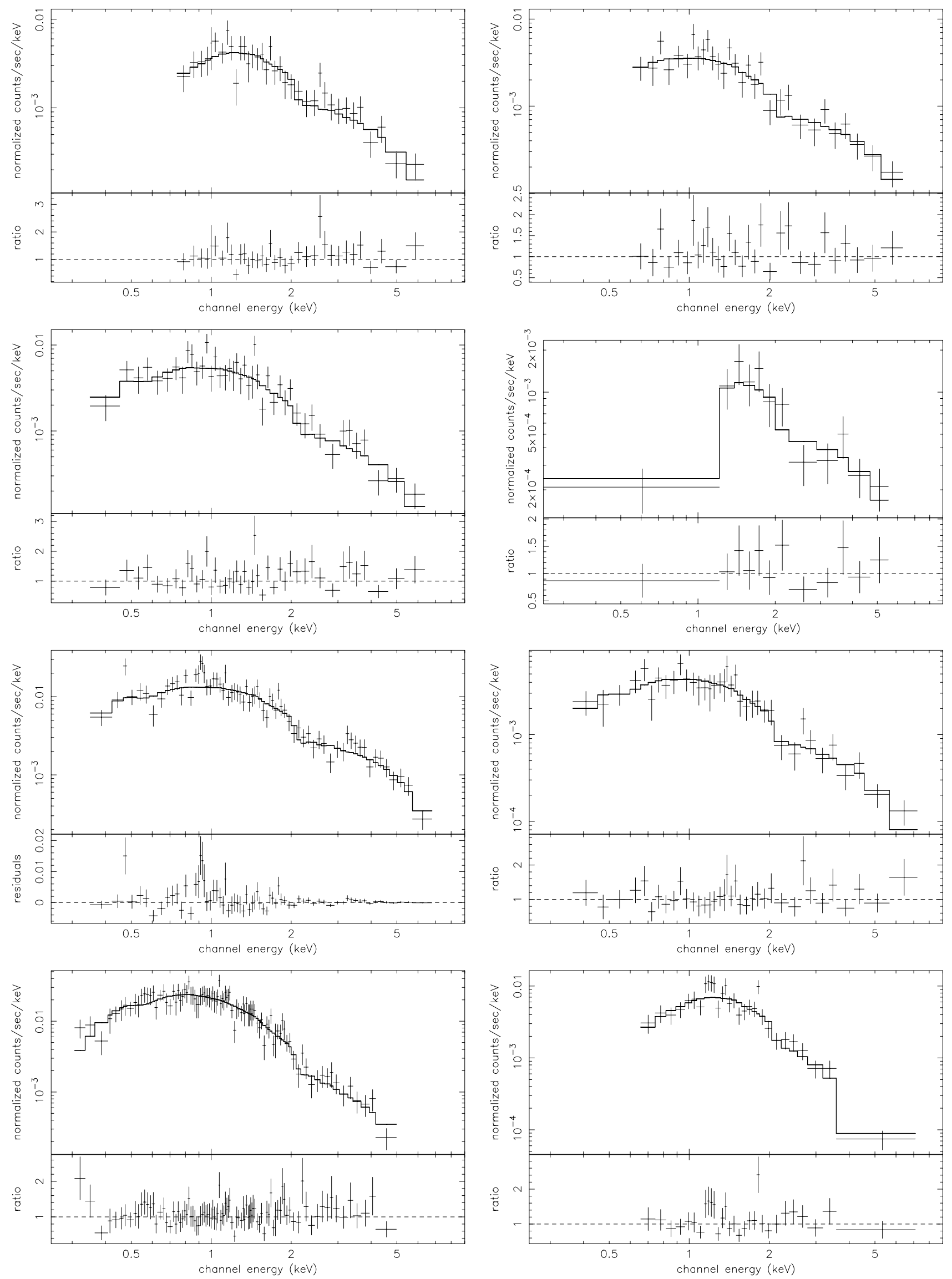

Fig. B.1. Spectra of bright sources of group C: from top left: No. 5 and 31; No. 33 (a candidate X-ray pulsar, lightcurve shown in Fig. 9) and 39 (a background radio galaxy); No. 44 and 60; No. 64 and 72. Their fitting parameters are listed in Table B.4. 

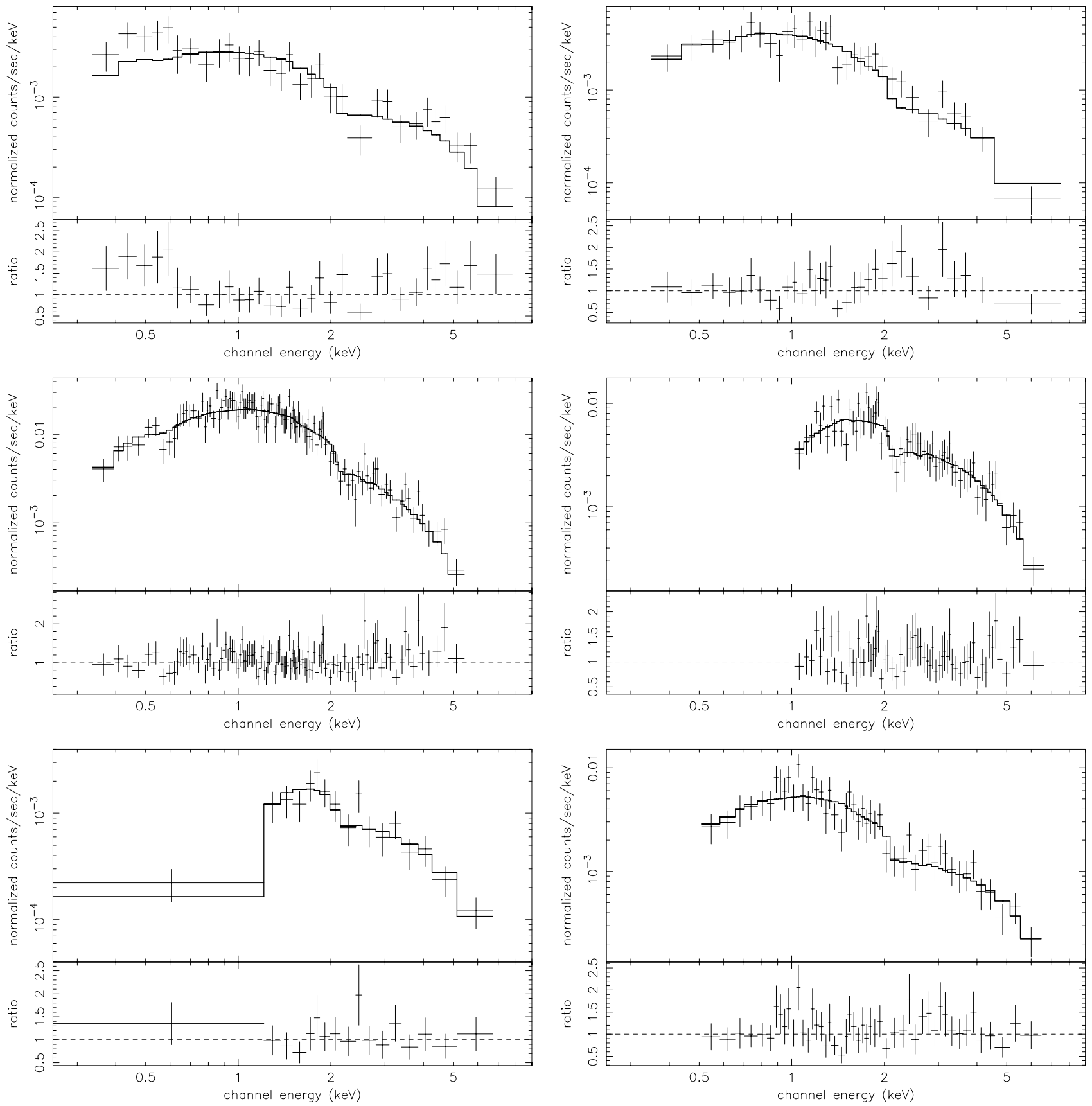

Fig. B.2. Spectra of bright sources of group C (continued): from top left: No. 78 and 85; No. 88 and 104. No. 113 (a candidate X-ray pulsar, lightcurve shown in Fig. 10) and No. 121. Their fitting parameters are listed in Table B.4. 


\section{References}

Arnaud, K. A. 1996, Astronomical Data Analysis Software and Systems V, ed. G. Jacoby, \& J. Barnes, ASP Conf. Ser., 101, 17

Blair, W. P., \& Long, K. S. 1997, ApJS, 108, 261

Blundell, K. M., Rawlings, S., \& Willott, C. J. 1999, AJ, 117, 677

Bohlin, R. C., Cornett, R. H., Hill, J. K., Smith, A. M., \& Stecher, T. P. 1983, ApJ, 274, L53

Buat, V., Boselli, A., Gavazzi, G., \& Bonfanti, C. 2002, A\&A, 383, 801

Chevalier, R. A., \& Fransson, C. 1994, ApJ, 420, 268

Corbet, R. H. D. 1986, MNRAS, 220, 1047

Corbet, R. H. D., Marshall, F. E., Coe, M. J., Laycock, S., \& Handler, G. 2001, ApJ, 548, L41

Côté, S., Freeman, K. C., Carignan, C., \& Quinn, P. 1997, AJ, 114, 1313

Cowan, J. J., \& Branch, D. 1985, ApJ, 293, 400

Cowan, J. J., Roberts, D. A., \& Branch, D. 1994, ApJ, 434, 128

de Vaucouleurs, G. 1979, AJ, 84, 1270

de Vaucouleurs, G., Pence, W. D., \& Davoust, E. 1983, ApJS, 53, 17

de Vaucouleurs, G., de Vaucouleurs, A., Corwin, H. Jr., et al. 1991, Third Reference Catalogue of Bright Galaxies (New York: Springer-Verlag)

Di Stefano, R., \& Kong, A. K. H. 2003, ApJ, 592, 884

Ehle, M., Pietsch, W., Beck, R., \& Klein, U. 1998, A\&A, 329, 39

Elmegreen, D. M., Chromey, F. R., \& Warren, A. R. 1998, AJ, 116, 2834

Fabbiano, G., Trinchieri, G., Elvis, M., Miller, L., \& Longair, M. 1984, ApJ, 277, 115

Fabbiano, G., Zezas, A., \& Murray, S. S. 2001, ApJ, 554, 1035

Gallais, P., Rouan, D., Lacombe, F., Tiphène, D., \& Vauglin, I. 1991, A\&A, 243, 309

Harris, J., Calizetti, D., Gallagher, J. S. III, Conselice, C. J., \& Smith, D. A. 2001, AJ, 122, 3046

Heckman, T. M. 1998, in the Proc. of the Conf. Origins, ed. C. E. Woodward, J. M. Shull, \& H. A. Thronson, Jr., ASP Conf. Ser., 148,127

Hughes, J. P. 1994, ApJ, 427, L25

Immler, S., Vogler, A., Ehle, M., \& Pietsch, W. 1999, A\&A, 352, 415

Kahabka, P. 1999, A\&A, 344, 459

Kembhavi, A. K., \& Narlikar, J. V. 1999, in Quasars and Active Galactic Nuclei (Cambridge: Cambridge University Press)

Kilgard, R. E., Kaaret, P., Krauss, M. I., et al. 2002, ApJ, 570, 671

Kong, A. K. H., Di Stefano, R., Garcia, M., \& Greiner, J. 2003, ApJ, 585, 298

La Barbera, A., Burderi, L., Di Salvo, T., Iaria, R., \& Robba, N. R. 2001, ApJ, 553, 375

Laycock, S., Corbet, R. H. D., Perrodin, D., et al. 2002, A\&A, 385, 464

Lewin, W. H. G., van Paradijs, J., \& van den Heuvel, E. P. J. 1995, $\mathrm{X}$-ray binaries (Cambridge: Cambridge University Press)

Ma, C., Arias, E. F., Eubanks, T. M., et al. 1998, AJ, 116, 516

Matsumoto, H., Tsuru, T. G., Koyama, K., et al. 2001, ApJ, 547, L25

Mavromatakis, F. 1993, A\&A, 273, 147

Negueruela, I. 1998, A\&A, 338, 505

Ohashi, T., Makishima, K., Tsuru, T., et al. 1990, ApJ, 365, 180

Okada, K., Mitsuda, K., \& Dotani, T. 1997, PASJ, 49, 653
Owen, F. N., \& Ledlow, M. J. 1994, Proc. of The First Stromlo Symp., The Physics of Active Galaxies, ed. G. V. Bicknell, M. A. Dopita, \& P. J. Quinn, ASP Conf. Ser., 54, 319

Pannuti, T. G. 2002, Ph.D. Thesis, available from http://space.mit.edu/ tpannuti

Pannuti, T. G., Duric, N., Lacey, C. K., et al. 2002, ApJ, 565, 966

Pooley, D., Lewin, W. H. G., Fox, D. W., et al. 2002, ApJ, 572, 932

Predehl, P., \& Schmitt, J. H. M. M. 1995, A\&A, 293, 889

Prestwich, A. H. 2001, Proc. of X-rays at Sharp Focus: Chandra Science Symp., ed. S. Vrtilek, E. M. Schlegel, \& E. Kuhi [astro-ph/0107133]

Prestwich, A. H., Irwin, J. A., Kilgard, R. E., et al. 2003, ApJ, in press [astro-ph/0206127]

Rappaport, S., Di Stefano, R., \& Smith, J. D. 1994, ApJ, 426, 692

Rosati, P., Tozzi, P., Giacconi, R., et al. 2002, ApJ, 566, 667

Sako, M., Liedahl, D. A., Kahn, S. M., \& Paerels, F. 1999, ApJ, 525, 921

Sandage, A., \& Tammann, G. A. 1987, A revised Shapley-Ames Catalog of Bright Galaxies, 2nd ed. (Washington: Carnegie Institution of Washington Publication)

Schlegel, D. E., Finkbeiner, D. P., \& Davis, M. 1998, ApJ, 500, 525

Shirey, R., Soria, R., Borozdin, K., et al. 2001, A\&A, 365, L195

Soria, R. 2002, Proc. of High Energy Processes and Phenomena in Astrophysics, IAU Symp. 214, Suzhou (China), 5-10 August 2002, ed. X. Li, Z. Wang, \& V. Trimble [astro-ph/0211024]

Soria, R., \& Wu, K. 2002, A\&A, 384, 99

Stockdale, C. J., Cowan, J. J., Maddox, L. A., et al. 2001, BAAS, 199, 1902

Sunyaev, R. 2001, Proc. of the ESO Workshop, Garching (Germany), 6-8 Sep. 1999 ed. L. Kaper, E. P. J. van der Heuvel, \& P. A. Woudt (Springer), 113

Sunyaev, R., \& Revnivtsev, M. 2000, A\&A, 358, 617

Supper, R., Hasinger, G., Lewin, W. H. G., et al. 2001, A\&A, 373, 63

Swartz, D. A., Ghosh, K. K., McCollough, M. L., et al. 2003, ApJS, 144,213

Swartz, D. A., Ghosh, K. K., Suleimanov, V., Tennant, A. F., \& Wu, K. 2002, ApJ, 574, 382

Talbot, R. J., Jensen, E. B., \& Dufour, R. J. 1979, ApJ, 229, 91

Tennant, A. F., Wu, K., Ghosh, K. K., Kolodziejcak, J. J., \& Swartz, D. A. 2001, ApJ, 549, L43

Thackeray, A. D. 1968, Sky \& Telescope, 36, 295

Thatte, N., Tecza, M., \& Genzel, R. 2000, A\&A, 364, L47

Tilanus, R. P. J., \& Allen, R. J. 1993, A\&A, 274, 707

Trinchieri, G., Fabbiano, G., \& Palumbo, C. G. C. 1985, ApJ, 290, 96 van den Bergh, S. 1980, PASP, 92, 122

van den Heuvel, E. P. J., Bhattacharya, D., Nomoto, K., \& Rappaport, S. A. 1992, A\&A, 262, 97

Wood, R., \& Andrews, P. J. 1974, MNRAS, 167, 13

Wu, K. 2001, Publ. Astron. Soc. Australia, 18, 443

Wu, K., Tennant, A. F., Swartz, D. A., Ghosh, K. K., \& Hunstead, R. W. 2003, Proc. of the Symp. New Visions of the X-ray Universe in the XMM-Newton and Chandra Era, ESTEC, The Netherlands, Nov. 2001 [astro-ph/0302363]

Zezas, A., Fabbiano, G., Rots, A. H., \& Murray, S. S. 2002, ApJS, 142,239 